\title{
Analytic Methods in Nonperturbative QCD
}

\author{
Yu.A.Simonov \\ State Research Center \\ Institute of Theoretical and Experimental Physics, \\ Moscow, Russia
}

\begin{abstract}
Recently developed analytic methods in the framework of the Field Correlator Method are reviewed in this series of four lectures and results of calculations are compared to lattice data and experiment. Recent lattice data demonstrating the Casimir scaling of static quark interaction strongly support the FCM and leave very little space for all other theoretical models, e.g. instatnton gas/liquid model. Results of calculations for mesons, baryons, quark-gluon plasma and phase transition temperature demonstrate that new analytic methods are a powerful tool of nonperturbative QCD along with lattice simulations.
\end{abstract}

\section{General QCD formalism for Green's functions.}

1. Starting to work in QCD one should have in mind some specific features, which make this theory unique; two of these are most important.

In QCD (as in any local gauge theory in the confining phase) it is impossible to introduce asymptotic states of quarks and gluons, hence the popular formalism of LSZ [1] is not applicable. Therefore one works with off-shell quantities, like the Green's functions and later on one introduces Hamiltonian and scattering amplitudes.

2. In QCD the Nonperturbative (NP) fields are Euclidean (c.f. [2]) and therefore one should work out the formalism of Euclidean vacuum averaging first, and later one goes over to the Minkowskian space (inevitable for scattering amplitudes.).

In this section we introduce first simple general and exact formulas for Green's functions, Hamiltonians etc. and later discuss possible approximations.

Consider a general partition function of QCD

$$
Z\left(J_{\mu}, \eta, \bar{\eta}\right)=\int D A_{\mu} D \psi D \psi^{+} e^{-S\left(A, \psi, \psi^{+}\right)+\int\left(J_{\mu} A_{\mu}+\eta^{+} \psi+\psi^{+} \eta\right) d^{4} x}
$$

where $S$ is the standard QCD Euclidean action.

$$
S=\frac{1}{2} \int t r F_{\mu \nu}^{2} d^{4} x-\int \psi^{+}(i \hat{\partial}+i m+g \hat{A}) d^{4} x
$$


Here gauge fixing terms and Faddeev-Popov ghosts are suppressed for simplicity.

A spicific feature of local gauge theories is that only gauge-invariants can enter as physical states, and local gauge invariance cannot be spontaneously violated (the Elitzur theorem). Therefore the only way to proceed is to form gauge-invariant Green's functions from (11). E.g. for the $\bar{q} q$ state one might have a local or nonlocal meson Green's function.

$$
G_{f, i n}^{(M)}(x x \mid y y)=\left\langle\psi^{+}(x) \Gamma_{f} \psi(x) \psi^{+}(y) \Gamma_{i n} \psi(y)\right\rangle, \quad \operatorname{or}\left\langle\psi^{+} \Gamma_{f} \Phi \psi \psi^{+} \Gamma_{i n} \Phi \psi\right\rangle
$$

where $\Gamma_{f}, \Gamma_{i n}=1, \gamma_{5}, \gamma_{\mu}, \ldots ; \Phi(x, y)=P \exp i g \int_{y}^{x} A_{\mu} d z_{\mu}$, and $\langle\ldots\rangle$ means averaging over $D A_{\mu} D \psi D \psi^{+}$as in (国).

Integrating over $D \psi D \psi^{+}$in (11) one obtains for the meson Green's function.

$$
\begin{gathered}
G^{(M)}=\int D A_{\mu} e^{-S_{0}(A)} \operatorname{det}(m+\hat{D})\left\{\operatorname{tr}_{\alpha}\left[\Gamma_{f} S(x, y) \Gamma_{i n} S(y, x)\right]-\right. \\
\left.-\operatorname{tr}_{\alpha}\left(S(x, x) \Gamma_{f}\right) \operatorname{tr}_{\alpha}\left(S(y, y) \Gamma_{i n}\right)\right\}
\end{gathered}
$$

with notatins for the quark Green's function

$$
S(x, y)=\left\langle x\left|(m+\hat{D})^{-1}\right| y\right\rangle .
$$

In QED one can define for $G^{(M)}$ the Bethe-Salpeter (BS) or similar equations to take into account the proper subclass of perturbative diagrams. In QCD this way is impossible since both BS and Dyson-Schwinger eqs are not gauge-invariant, whenever one approximates the kernels by finite sets of diagrams.

For gluons one should introduce gauge-invariant initial and final states made of $F_{\mu \nu}, D_{\mu}$. E.g. for local states $\Psi_{f(i n)}=\operatorname{tr} F_{\mu \nu}(x) F_{\alpha \beta}(x)$ or $\left.\operatorname{tr} D_{\rho} F_{\mu \nu}(x) F_{\alpha \beta}(x)\right)$ etc. and define Green's functions

$$
G_{f, i n}^{(G l)}=\left\langle\Psi_{f}^{+}(x) \Psi_{i n}(y)\right\rangle .
$$

Integration over $D \psi D \psi^{+}$allows to obtain only the form

$$
G_{f, i n}^{(G l)}=\int D A_{\mu} e^{-S_{0}(A)} \operatorname{det}(m+\hat{D}) \Psi_{f}^{+}(x) \Psi_{i n}(y) .
$$

To proceed one should use the Background Field Formalism (BFF) and the Feynman-Schwinger (or world-line) Representation (FSR) which will be discussed in the next lectures.

Here we shall give connection to the Hamiltonian technique, which is useful both for bound states and for scattering.

The following general relation holds true for any Green's function

$$
G_{f, i n}^{(k)}\left(x, y \mid x^{\prime}, y^{\prime}\right)=\left\langle f, x, y\left|e^{-H T}\right| i n, x^{\prime}, y^{\prime}\right\rangle=\left\langle f, x, y\left|n>e^{-M_{n} T}<n\right| i n, x^{\prime}, y^{\prime}\right\rangle
$$

with $M_{n}, \mid n>$ being eigenvalues and eigenfunctions of $H$ respectively. Here we have defined a set of hypersurfaces $\left\{\sum_{t}\right\}$ on which the Hamiltonian is constructed, and 
the evolution parameter $t$, perpendicular to $\sum_{t}$, which varies between, say, $t=0$ for the in-state and $t=T$ for the final state.

The Hamiltonian $H$ is the general QCD Hamiltonian which is known in several gauges, e.g. Coulomb [3], temporal [4] or in polar representation [5]. It is a matrix, containing creation and destruction of quarks and gluons, and angular brackets in (8) do single out the initial and final indices of this matrix.

The most important feature of QCD is confinement, which means that some part of gluons are condensed in the form of strings between charges. Unfortunately the general QCD Hamiltonians [3]-[5] are not yet helpful in understanding confinement properties of QCD, and one cannot use it as a tool in exploring vacuum properties.

Therefore our main aim would be to formulate effective Hamiltonian for the valence (noncondensed) gluons and quarks.

The way to do it will be described in next lectures.

\section{Background Field Formalism}

One of basic mysteries of QCD is the fact, that gluon field plays two different roles:

i) gluons are propagating, and at small distances this process can be described perturbatively, leading in particular to color Coulomb interaction between quarks (antiquarks);

ii) gluons form a kind of condensate, which serves as a background for the propagating perturbative gluons and quarks. This background is Euclidean and ensures phenomena of confinement and CSB.

Correspondingly we shall separate the total gluonic field $A_{\mu}$ into perturbative part $a_{\mu}$ and nonperturbative (NP) background $B_{\mu}$ :

$$
A_{\mu}=B_{\mu}+a_{\mu}
$$

There are many questions about this separation, which may be answered now only partially, e.g. what exactly is the criterion of separation. Possible answer is that perturbative fields $a_{\mu}$ get their dimension from distance (momentum), and therefore all correlators of fields $a_{\mu}$ (in absence of $B_{\mu}$ ) are singular and made of inverse powers of $(x-y)$ and logarithms, where enters the only dimensional parameter of perturbative QCD $-\Lambda_{Q C D}$. Therefore evidently any dimensionful constant, like hadronic masses or string tension cannot be obtained as a perturbation series. In contrast to that, NP fields $B_{\mu}$ have mass dimension due to the violation of scale invariance which is intrinsically present in the gluodynamics Lagrangian. The origin of separation (9)) is clearly seen in the solutions of nonlinear equations for field correlators [6]: a perturbative solution of those leads to singular power-like field correlator, whereas at large distances there is a selfconsistent solution of the equations, decaying exponentially with distance with arbitrary mass scale, since equations in [6] are scale-invariant. Full solution including intermediate distances produces mixed 
perturbative-nonperturbative terms, containing both inverse powers of distance and exponentials. For these terms criterion of separation fails.

One can avoid formally the question of separation principle (and of double counting) using t'Hooft identity [7], which allows to integrate in (1) independently over $B_{\mu}$ and $a_{\mu}$ :

$$
Z=\frac{1}{N^{\prime}} \int D B_{\mu} \eta(B) D \psi D \bar{\psi} D a_{\mu} e^{L(B+a)}
$$

Here $L(A)=L_{0}+L_{1}+L_{i n t}$, and $A_{\mu}$ is taken to be $B_{\mu}+a_{\mu}$; the weight $\eta(B)$ is arbitrary and may be taken as constant. For the exact formalism starting from (10) we refer the reader to [7, 8], and here we only quote the form of background propagator $G_{\mu \nu}$ of the gluon, which is found from $L(B+a)$ and will be used in what follows,

$$
G_{\mu \nu}=\left(D_{\lambda}^{2} \delta_{\mu \nu}+2 i g F_{\mu \nu}\right)^{-1}
$$

where $D_{\lambda}=\partial_{\lambda}-i g B_{\lambda}$, and $F_{\mu \nu}=\partial_{\mu} B_{\nu}-\partial_{\nu} B_{\mu}-i g\left[B_{\mu}, B_{\nu}\right]$.

To define the perturbation theory series in $g a_{\mu}$ one starts from (10) and rewrites the Lagrangian as follows:

$$
\begin{gathered}
L_{t o t}=L_{g f}+L_{g h}+L(B+a)= \\
L_{0}+L_{1}+L_{2}+L_{i n t}+L_{g f}+L_{g h}
\end{gathered}
$$

where $L_{i}$ have the form:

$$
\begin{gathered}
L_{2}(a)=\frac{1}{2} a_{\nu}\left(\hat{D}_{\lambda}^{2} \delta_{\mu \nu}-\hat{D}_{\mu} \hat{D}_{\nu}+i g \hat{F}_{\mu \nu}\right) a_{\mu}= \\
=\frac{1}{2} a_{\nu}^{c}\left[D_{\lambda}^{c a} D_{\lambda}^{a d} \delta_{\mu \nu}-D_{\mu}^{c a} D_{\nu}^{a d}-g f^{c a d} F_{\mu \nu}^{a}\right] a_{\mu}^{d}, \\
D_{\lambda}^{c a}=\partial_{\lambda} \cdot \delta_{c a}+g f^{c b a} B_{\lambda}^{b} \equiv \hat{D}_{\lambda} \\
L_{0}=-\frac{1}{4}\left(F_{\mu \nu}^{a}(B)\right)^{2} ; \quad L_{1}=a_{\nu}^{c} D_{\mu}^{c a}(B) F_{\mu \nu}^{a} \\
L_{i n t}=-\frac{1}{2}\left(D_{\mu}(B) a_{\nu}-D_{\nu}(B) a_{\mu}\right)^{a} g f^{a b c} a_{\mu}^{b} a_{\nu}^{c}-\frac{1}{4} g^{2} f^{a b c} a_{\mu}^{b} a_{\nu}^{c} f^{a e f} a_{\mu}^{e} a_{\nu}^{f} \\
L_{\text {ghost }}=-\theta_{a}^{+}\left(D_{\mu}(B) D_{\mu}(B+a)\right)_{a b} \theta_{b} .
\end{gathered}
$$

To calculate Green's functions of any hadrons one can use (10) and write

$$
\begin{gathered}
G_{h}(X ; Y)=\mathrm{const} \int D B_{\mu} \eta(B) D \psi D \bar{\psi} D a_{\mu} \Psi_{f}^{+}(X) \Psi_{i n}(Y) e^{L_{t o t}} \\
\equiv\left\langle\Psi_{f}^{+}(X) \Psi_{i n}(Y)\right\rangle_{B, \psi, a} .
\end{gathered}
$$

Here $\Psi_{f, i n}$ are initial and final hadron states made of $B, \psi, \bar{\psi}, a$. To calculate the integral $D a_{\mu}$ one can use perturbative expansion in $g a_{\mu}$, i.e. neglect in first approximation $L_{\text {int }}$, containing terms $a^{3}, a^{4}$ and take into account only quadratic terms 
$L_{2}, L_{g f}, L_{g h}$. The contribution of $L_{1}$ was studied in [8] and it was shown there that for the most processes (e.g. for hadron Green's functions) it can be neglected (with accuracy of about better than $10 \%$ ).

It is convenient to choose background gauge for $a_{\mu}, D_{\mu} a_{\mu}=0$, and take gauge transformation in the form

$$
B_{\mu} \rightarrow U^{+}\left(B_{\mu}+\frac{i}{g} \partial_{\mu}\right) U, a_{\mu} \rightarrow U^{+} a_{\mu} U
$$

Now one can choose $\Psi_{f}, \Psi_{i n}$ as gauge-invariant forms built from $B_{\mu}, a_{\mu}, \psi, \bar{\psi}$ and to average over $B_{\mu}, \psi, \bar{\psi}, a_{\mu}$ as shown in (16). Integrating over $D B_{\mu}$ might seem an impossible adventure, but we shall see that it can be always written as products of Wilson loops with some insertions, which can be treated in two ways:

i) or in the form of the area law. Then contribution of fields $B_{\mu}$ is given and fixed by the string tension $\sigma(B)$. Equating $\sigma(B)=\sigma_{\text {exper }}$ one fixes contribution of the background fields.

ii) Using cluster expansion, and expressing result through lowest correlators, e.g. $\langle F F\rangle$, or its scalar parts $D(x)$ and $D_{1}(x)$. In this case background is fixed by these functions taken as an input, e.g. from lattice data.

The rest integrals, over $D a_{\mu} D \psi D \bar{\psi}$ are Gaussian and can be simply done. Now we turn to the construction of $\Psi_{f, i n}$.

One can choose local or nonlocal forms for $\Psi_{i n, f}$, the latter are obtained by insertion of $\Phi(x, y)$ in the local expressions, which are

$\Psi_{i n, f}=\bar{\psi} \Gamma_{i} \psi$ for mesons, $\Gamma_{i}=1, \gamma_{5}, \gamma_{\mu}, \gamma_{\mu} \gamma_{5}, \sigma_{\mu \nu}$

$\Psi_{i n, f}=\bar{\psi} \Gamma_{i} f(a, D a) \psi$ for hybrids, where $f$ is a polynomial, the simplest form is $\bar{\psi} \gamma_{i} a_{\mu} \psi$

$\Psi_{i n, f}=\operatorname{tra} \Lambda_{2} a$ and $\operatorname{tr}\left(\Lambda_{3} a^{3}\right)$ for glueballs made of 2 and 3 gluons respectively. $\Lambda_{2}, \Lambda_{3}$ are polynomials made of $D_{\mu}(B)$ and ensure the needed tensor structure for given quantum numbers. In the simplest case $\Lambda_{2}=\Lambda_{3}=1$.

$\Psi_{i n, f}=e_{a b c} \psi_{f_{1} \alpha}^{a} \psi_{f_{2} \beta}^{b} \psi_{f_{3} \gamma}^{c} K^{f_{1} \alpha, f_{2} \beta, f_{3} \gamma}$ for baryons, where $a, f_{i}, \alpha$ are color, flavour and Lorentz index respectively.

It is clear that higher hybrid states for mesons and baryons are obtained by adding factors of $a_{\mu}$ in $\Psi_{i n, f}$.

Insertion of given $\Psi_{i n, f}$ in (16) yields after integration over $D a_{\mu} D \psi D \bar{\psi}$ the hadron Green's function, which can be written respectively for mesons

$$
\begin{gathered}
G_{M}(X, Y)=\left\langle\operatorname{tr}\left(\Gamma_{i}^{(f)}(X) G_{q}(X, Y) \Gamma_{k}^{(i n)}(Y) G_{\bar{q}}(X, Y)\right)\right\rangle_{B} \\
-\left\langle\operatorname{tr}\left(\Gamma_{i}^{(f)}(X) G_{q}(X, X)\right) \operatorname{tr}\left(\Gamma_{k}^{(i n)}(Y) G_{\bar{q}}(Y, Y)\right)\right\rangle_{B},
\end{gathered}
$$

where $G_{q, \bar{q}}$ is the quark (antiquark) Green's function,

$$
G_{q}(X, Y)=(\hat{\partial}+m-i g \hat{B})_{X, Y}^{-1},
$$

for hybrids

$$
G_{h y b}(X, Y)=\left\langle\operatorname{tr}\left(\Gamma_{i}^{(f)}(X) G_{q}(X, Y) G_{g}(X, Y) \Gamma_{k}^{(i n)} G_{\bar{q}}(X, Y)\right)\right\rangle_{B},
$$


where $G_{g}$ is the gluon Green's function (11).

For glueballs one has

$$
G_{\text {glueball }}=\left\langle\operatorname{tr}\left(\Lambda_{2}^{(f)}(X) G_{g}(X, Y) \Lambda_{2}^{(i n)}(Y) G_{g}(X, Y)\right)\right\rangle_{B}
$$

and for baryons

$$
G_{B}(X, Y)=\left\langle\operatorname{tr}\left(e K^{(f)} \prod_{i=1}^{3} G_{q}^{(i)}(X, Y) e K^{(i n)}\right)\right\rangle_{B}
$$

The structure of hadron Green's function is clear: it is a product of quark and gluon Green's function averaged over the background field $B_{\mu}$. In the next section we shall discuss the way, how to make this averaging explicit and express it through the Wilson loop.

\section{Feynman-Schwinger representation}

The present stage of development of field theory in general and of QCD in particular requires the exploiting of nonperturbative methods in addition to summing up perturbative series. This calls for specific methods where dependence on vacuum fields can be made simple and explicit. A good example is provided by the so-called Feynman-Schwinger Representation (FSR) 9, 10] based on the Schwinger proper time and Feynman path integral formalism [11, 12].

For some earlier references see [10] and [13]. More recently some modification of FSR was exploited in [14 and [15]. The double-logarithm perturbative summation of amplitudes is especially convenient for FSR and was extensively studied in [16].

Meanwhile the first extension of FSR to nonzero temperature field theory was done in [17]. That was the basis for the systematic study of the role of nonperturbative (NP) configurations in the temperature phase transitions [18, 19, 20, 21].

One of the most important advantages of FSR is that it allows to reduce physical amplitudes to the weighted integrals of the averaged Wilson loops. Thus the field (both perturbative and NP) enter only through Wilson loops and for the latter one can apply cluster expansion theorem [22] which allows to sum up a series of approximations directly in the exponent.

Therefore one can avoid Feynman diagram summation to get asymptotics of formfactors, as it was done in [16].

The role of FSR in the treatment of NP effects is more crucial. In this case one can develop a powerful method of Background Perturbation Theory treating NP fields as a background [7, 8].

We start with the scalar particle (e.g. Higgs boson) interacting with the nonabelian vector potential via Euclidean Lagrangian

$$
L_{\varphi}=\left|D_{\mu} \varphi\right|^{2} \equiv\left|\left(\partial_{\mu}-i g A_{\mu}\right) \varphi\right|^{2}
$$


so that the Green's function of $\varphi$ can be written using the Fock-Schwinger proper time representation as

$$
G(x, y)=\left(m^{2}-D_{\mu}^{2}\right)_{x y}^{-1}=\left\langle x\left|P \int_{0}^{\infty} d s e^{-s\left(m-D_{\mu}^{2}\right)}\right| y\right\rangle
$$

The next step can be done as in [9], treating $s$ as the ordering parameter. Note the difference of the integral (24) from the case of QED treated in [10, 12, 13]: $A_{\mu}$ in our case is the matrix operator $A_{\mu}(x)=A_{\mu}^{a}(x) T^{a}$ and it does not commute for different $x$, hence the ordering operator $P$ in (24). The exact meaning of $P$ becomes clear in the final form of the path integral

$$
G(x, y)=\int_{0}^{\infty} d s(D z)_{x y} e^{-K} P \exp \left(i g \int_{y}^{x} A_{\mu}(z) d z_{\mu}\right)
$$

where $K=\frac{1}{4} \int_{0}^{s} d \tau\left(\frac{d z_{\mu}}{d \tau}\right)^{2}+m^{2} s$, and

$$
(D z)_{x y}=\lim _{N \rightarrow \infty} \prod_{n=1}^{N} \int \frac{d^{4} z(n)}{(4 \pi \varepsilon)^{2}} \int \frac{d^{4} p}{(2 \pi)^{4}} e^{i p\left(\sum_{n=1}^{N} z(n)-(x-y)\right)}, \quad N \varepsilon=s .
$$

The last integral in (26) ensures that the path $z_{\mu}(\tau), 0 \leq \tau \leq s$, starts at $z_{\mu}(0)=y_{\mu}$ and ends at $z_{\mu}(s)=x_{\mu}$ The form of (25) is the same as in the case of QED except for the ordering operator $P$ which gives exact meaning to the integral of the noncommuting matrices $A_{\mu_{1}}\left(z_{1}\right), A_{\mu_{2}}\left(z_{2}\right)$ etc. In the case of QCD the form (25), (26) was introduced in [9].

The usefulness of the FSR (25) becomes clear when one considers the physical amplitude, e.g. the Green's function of the white state $\operatorname{tr}\left(\varphi^{+}(x) \varphi(x)\right)$ or its nonlocal version $\operatorname{tr}\left[\varphi^{+}(x) \Phi(x, y) \varphi(y)\right]$, where $\Phi(x, y)$ to be widely used in what follows is parallel transporter along some arbitrary contour $C(x, y)$

$$
\Phi(x, y)=P \exp \left(i g \int_{y}^{x} A_{\mu}(z) d z_{\mu}\right)
$$

One has by standard rules

$$
\begin{gathered}
G_{\varphi}(x, y)=\left\langle\operatorname{tr}\left(\varphi^{+}(x) \varphi(x)\right) \operatorname{tr}\left(\varphi^{+}(y) \varphi(y)\right)\right\rangle_{A}= \\
=\int_{0}^{\infty} d s_{1} \int_{0}^{\infty} d s_{2}(D z)_{x y}\left(D z^{\prime}\right)_{x y} e^{-K-K^{\prime}}\langle W\rangle_{A}+\ldots
\end{gathered}
$$

where dots stand for the disconnected part, $\left\langle G_{\varphi}(x, x) G_{\varphi}(y, y)\right\rangle_{A}$ and we have used the fact that the propagator of the charge-conjugated particle $\varphi^{+}$is proportional to

$$
\Phi_{C}(x, y)=P \exp \left(i g \int_{y}^{x} A_{\mu}^{(C)}(z) d z_{\mu}\right), \quad A_{\mu}^{(C)}=-A_{\mu}^{(T)}
$$

and therefore the ordering $P$ must be inverted, $\Phi_{C}(x, y)=P^{(T)} \exp \left(i g \int_{x}^{y} A_{\mu}(z) d z_{\mu}\right)$. 
Thus all dependence on $A_{\mu}(x)$ in $G_{\varphi}$ is reduced to the Wilson loop average

$$
\langle W\rangle_{A}=\left\langle\operatorname{tr} P_{C} \exp i g \int_{C} A_{\mu}(z) d z_{\mu}\right\rangle_{A} .
$$

Here $P_{C}$ is the ordering around the closed loop $C$ passing through the points $x, y$; the loop being made of the path $z_{\mu}(\tau)$ and $z_{\mu}^{\prime}\left(\tau^{\prime}\right)$ to be integrated over in the integral (28).

Consider now a fermion (quark) Green's function in the Euclidean external gluonic field. Similarly to the QED case considered in [10 one has

$$
\begin{gathered}
G_{q}(x, y)=\langle\psi(x) \bar{\psi}(y)\rangle_{q}=\left\langle x\left|\left(m_{q}+\hat{D}\right)^{-1}\right| y\right\rangle= \\
=\left\langle x\left|\left(m_{q}-\hat{D}\right)\left(m_{q}^{2}-\hat{D}^{2}\right)^{-1}\right| y\right\rangle= \\
\left(m_{q}-\hat{D}\right) \int_{0}^{\infty} d s(D z)_{x y} e^{-K} \Phi_{\sigma}(x, y)
\end{gathered}
$$

where $\Phi_{\sigma}$ is the same as was introduced in [9, 23],

$$
\begin{aligned}
\Phi_{\sigma}(x, y) & =P_{A} P_{F} \exp \left(i g \int_{y}^{x} A_{\mu} d z_{\mu}\right) \times \\
& \times \exp \left(g \int_{0}^{s} d \tau \sigma_{\mu \nu} F_{\mu \nu}\right)
\end{aligned}
$$

and $\sigma_{\mu \nu}=\frac{1}{4 i}\left(\gamma_{\mu} \gamma_{\nu}-\gamma_{\nu} \gamma_{\mu}\right)$, while $K$ and $(D z)_{x y}$ are defined in (25) and (26). Note that operators $P_{A}, P_{F}$ in (32) preserve the proper ordering of matrices $A_{\mu}$ and $\sigma_{\mu \nu} F_{\mu \nu}$ respectively; the explicit examples will be considered below.

Finally we come to the case of FSR for the valence gluon propagating in the background nonabelian field; the detailed study of this case is given in chapter 5; here we only quote the result (11) for the gluon Green's function in the background Feynman gauge

$$
G_{\mu \nu}(x, y)=\left\langle x\left|\left(D_{\lambda}^{2} \delta_{\mu \nu}+2 i g F_{\mu \nu}\right)^{-1}\right| y\right\rangle, D_{\lambda}=\partial_{\lambda}-i g B \lambda .
$$

Proceeding in the same way as for quarks, one obtains the FSR for the gluon Green's function [8]

$$
G_{\mu \nu}(x, y)=\int_{0}^{\infty} d s(D z)_{x y} e^{-K_{0}} \Phi_{\mu \nu}(x, y)
$$

where we have defined

$$
\begin{gathered}
K_{0}=\frac{1}{4} \int_{0}^{\infty}\left(\frac{d z_{\mu}}{d \tau}\right)^{2} d \tau ; \quad \Phi_{\mu \nu}(x, y)=\left[P_{F} P_{A} \exp \left(i g \int_{y}^{x} A_{\lambda} d z_{\lambda}\right) \times\right. \\
\left.\times \exp \left(2 i g \int_{0}^{s} d \tau F_{\sigma \rho}(z(\tau))\right)\right]_{\mu \nu}
\end{gathered}
$$

Now in the same way as it was done above for scalars in (28), one can consider a Green's function for the physical transition amplitude from a white state of $q_{1}, \bar{q}_{2}$ to another white state consisting of $q_{3}, \bar{q}_{4}$, i.e the meson Green's function.

$$
G_{q \bar{q}}(x, y)=\left\langle G_{q}(x, y) \Gamma G_{\bar{q}}(x, y)-G_{q}(x, x) \Gamma G_{\bar{q}}(y, y)\right\rangle_{A}
$$

and the first term on the r.h.s. of (36) can be reduced to the same form as in (28) but with the Wilson loop containing ordered insertions of the operators $\sigma_{\mu \nu} F_{\mu \nu}$ (cf.(32)). 


\section{$4 \quad$ FSR at nonzero temperature}

This part of lecture is based on papers 17, 18, where FSR was first introduced for $T>0$. We discuss first basic formalism at $T>0$ and then gluon and quark Green's functions.

\subsection{Basic equations}

We start with standard formulas of the background field formalism [7, 8] generalized to the case of nonzero temperature. We assume that the gluonic field $A_{\mu}$ can be split into the background field $B_{\mu}$ and the quantum field $a_{\mu}$

$$
A_{\mu}=B_{\mu}+a_{\mu}
$$

both satisfying periodic boundary conditions

$$
\begin{aligned}
& B_{\mu}\left(z_{4}, z_{i}\right)=B_{\mu}\left(z_{4}+n \beta, z_{i}\right), \\
& a_{\mu}\left(z_{4}, z_{i}\right)=a_{\mu}\left(z_{4}+n \beta, z_{i}\right),
\end{aligned}
$$

where $n$ is an integer and $\beta=1 / T$. The partition function can be written as

$$
\begin{gathered}
Z(V, T, \mu=0)=\langle Z(B)\rangle_{B}, \\
Z(B)=N \int D \phi \exp \left(-\int_{0}^{\beta} d \tau \int d^{3} x L_{t o t}(x, \tau)\right),
\end{gathered}
$$

where $\phi$ denotes all set of fields $a_{\mu}, \Psi, \Psi^{+}, N$ is normalization constant, and the sign \langle\rangle$_{B}$ means some averaging over (nonperturbative) background fields $B_{\mu}$, the exact form of this averaging is not needed for our purposes. Here $L_{t o t}$ is the same as in (12)-(15).

Integration over ghost and gluon degrees of freedom in (39) yields

$$
\begin{aligned}
& Z(B)=N^{\prime}(\operatorname{det} W(B))_{r e d}^{-1 / 2}\left[\operatorname{det}\left(-D_{\mu}(B) D_{\mu}(B+a)\right)\right]_{a=\frac{\delta}{\delta J}} \times \\
& \times\left\{1+\sum_{l=1}^{\infty} \frac{S_{\text {int }}^{l}}{l !}\left(a=\frac{\delta}{\delta J}\right)\right\} \exp \left(-\frac{1}{2} J W^{-1} J\right)_{J_{\mu}=D_{\mu}(B) F_{\mu \nu}(B)} .
\end{aligned}
$$

One can consider strong background fields, so that $g B_{\mu}$ is large (as compared to $\Lambda_{Q C D}^{2}$ ), while $\alpha_{s}=g^{2} / 4 \pi$ in that strong background is small at all distances. Moreover it was shown that $\alpha_{s}$ is frozen at large distances [7, 8, 25, 26].

In this case (40) is a perturbative sum in powers of $g^{n}$, arising from expansion in $\left(g a_{\mu}\right)^{n}$.

In what follows we shall discuss the Feynman graphs for the free energy $F(T)$, connected to $Z(B)$ via

$$
F(T)=-T \ln \langle Z(B)\rangle_{B}
$$

As will be seen, the lowest order graphs already contain a nontrivial dynamical mechanism for the deconfinement transition, and those will be considered in the next section. 


\subsection{The lowest order gluon contribution}

To the lowest order in $g a_{\mu}$ (and keeping all dependence on $g B_{\mu}$ explicitly) one has

$$
Z_{0}=e^{-F_{0}(T) / T}=N^{\prime}\left\langle\exp \left(-F_{0}(B) / T\right)\right\rangle_{B},
$$

where using (40) $F_{0}(B)$ can be written as

$$
\begin{gathered}
\frac{1}{T} F_{0}(B)=\frac{1}{2} \ln \operatorname{det} W-\ln \operatorname{det}\left(-D^{2}(B)\right)= \\
=S p\left\{-\frac{1}{2} \int_{0}^{\infty} \zeta(t) \frac{d t}{t} e^{-t W}+\int_{0}^{\infty} \zeta(t) \frac{d t}{t} e^{t D^{2}(B)}\right\} .
\end{gathered}
$$

In (43) $S p$ implies summing over all variables (Lorentz and color indices and coordinates), $\zeta(t)=\left.\lim \frac{d}{d s} \frac{M^{2 s} t^{s}}{\Gamma(s)}\right|_{s=0}$ is regularizing factor, one can use also the PauliVillars form for $\zeta(t)$.

Graphically, the first term on the r.h.s. of (43) and is a gluon loop in the background field, the second term is a ghost loop.

Let us turn now to the averaging procedure in (42). With the notation $\varphi=$ $-F_{0}(B) / T$, one can exploit in (42) the cluster expansion [22]

$$
\langle\exp \varphi\rangle_{B}=\exp \left(\sum_{n=1}^{\infty}\left\langle\left\langle\varphi^{n}\right\rangle\right\rangle \frac{1}{n !}\right)=\exp \left\{\langle\varphi\rangle_{B}+\frac{1}{2}\left[\langle\varphi\rangle_{B}^{2}-\left\langle\varphi^{2}\right\rangle_{B}\right]+O\left(\varphi^{3}\right)\right\} .
$$

To get a closer look at $\langle\varphi\rangle_{B}$ we first should discuss thermal propagators of gluon and ghost in the background field. We start with the thermal ghost propagator and write the FSR for it [8]

$$
\left(-D^{2}\right)_{x y}^{-1}=\left\langle x\left|\int_{0}^{\infty} d t e^{t D^{2}(B)}\right| y\right\rangle=\int_{0}^{\infty} d t(D z)_{x y}^{w} e^{-K} \hat{\Phi}(x, y) .
$$

Here $\hat{\Phi}$ is the parallel transporter in the adjoint representation along the trajectory of the ghost:

$$
\hat{\Phi}(x, y)=P \exp \left(i g \int \hat{B}_{\mu}(z) d z_{\mu}\right)
$$

also

$$
K=\frac{1}{4} \int_{0}^{t} d \tau \dot{z}_{\mu}^{2} ; \quad \dot{z}_{\mu}=\frac{\partial z_{\mu}(\tau)}{\partial \tau}
$$

and $(D z)_{x y}^{w}$ is a path integration with boundary conditions imbedded (this is marked by the subscript $(x y))$ and with all possible windings in Euclidean temporal direction (marked by the superscript $w$ ).

One can write it explicitly as

$$
(D z)_{x y}^{w}=\lim _{N \rightarrow \infty} \prod_{m=1}^{N} \frac{d^{4} \zeta(m)}{(4 \pi \varepsilon)^{2}} \sum_{n=0, \pm, \ldots} \frac{d^{4} p}{(2 \pi)^{4}} \exp \left[i p\left(\sum_{m=1}^{N} \zeta(m)-(x-y)-n \beta \delta_{\mu 4}\right)\right] .
$$


Here, $\zeta(k)=z(k)-z(k-1), \quad N \varepsilon=t$.

One check that in the free case, $\hat{B}_{\mu}=0$ (45) reduces to well-known form of the free propagator

$$
\begin{gathered}
\left(-\partial^{2}\right)_{x y}^{-1}=\int_{0}^{\infty} d t \exp \left[-\sum_{1}^{N} \frac{\zeta^{2}(m)}{4 \varepsilon}\right] \prod_{m} \overline{d \zeta(m)} \sum_{n} \frac{d^{4} p}{(2 \pi)^{4}} \times \\
\quad \times \exp \left[i p\left(\sum \zeta(m)-(x-y)-n \beta \delta_{\mu 4}\right)\right]= \\
=\sum_{n} \int_{0}^{\infty} \exp \left[-p^{2} t-i p(x-z)-i p_{4} n \beta\right] d t \frac{d^{4} p}{(2 \pi)^{4}}
\end{gathered}
$$

with

$$
\overline{d \zeta(m)} \equiv \frac{d \zeta(m)}{(4 \pi \varepsilon)^{2}}
$$

Using the Poisson summation formula

$$
\frac{1}{2 \pi} \sum_{n=0, \pm 1, \pm 2 \ldots} \exp \left(i p_{4} n \beta\right)=\sum_{k=0, \pm 1, \ldots} \delta\left(p_{4} \beta-2 \pi k\right)
$$

one finally gets the standard form

$$
\left(-\partial^{2}\right)_{x y}^{-1}=\sum_{k=0, \pm 1, \ldots} \int \frac{T d^{3} p}{(2 \pi)^{3}} \frac{\exp \left[-i p_{i}(x-y)_{i}-i 2 \pi k T\left(x_{4}-y_{4}\right)\right]}{p_{i}^{2}+(2 \pi k T)^{2}} .
$$

Note that, as expected, the propagator (45),(50) corresponds to a sum of ghost paths with all possible windings around the torus. The momentum integration in (47) asserts that the sum of all infinitesimal "walks" $\zeta(m)$ should be equal to the distance $(x-y)$ modulo $N$ windings in the compactified fourth coordinate.

For the gluon propagator in the background gauge we obtain similarly to (45)

$$
(W)_{x y}^{-1}=\int_{0}^{\infty} d t(D z)_{x y}^{w} e^{-K} \hat{\Phi}_{F}(x, y)
$$

where

$$
\hat{\Phi}_{F}(x, y)=P_{F} P \exp \left(2 i g \int_{0}^{t} \hat{F}(z(\tau)) d \tau\right) \exp \left(i g \int_{y}^{x} \hat{B}_{\mu} d z_{\mu}\right)
$$

and the operators $P_{F} P$ are used to order insertions of $\hat{F}$ on the trajectory of the gluon.

Now we come back to the first term in (44), $\langle\varphi\rangle_{B}$, which can be represented with the help of (45) and (51) as

$$
\langle\varphi\rangle_{B}=\int \frac{d t}{t} \zeta(t) d^{4} x(D z)_{x x}^{w} e^{-K}\left[\frac{1}{2} \operatorname{tr}\left\langle\hat{\Phi}_{F}(x, x)\right\rangle_{B}-\langle\operatorname{tr} \hat{\Phi}(x, x)\rangle_{B}\right],
$$

where the sign $t r$ implies summation over Lorentz and color indices.

One can easily show (see [8] for details) that (53) yields for $B_{\mu}=0$ the usual result for the free gluon gas:

$$
F_{0}(B=0)=-T \varphi(B=0)=-\left(N_{c}^{2}-1\right) V_{3} \frac{T^{4} \pi^{2}}{45} .
$$




\subsection{The lowest order quark contribution}

Integrating over quark fields in (39) is done trivially and leads to the following

additional factor in (40)

$$
\operatorname{det}(m+\hat{D}(B+a))=\frac{1}{2} \operatorname{det}\left(m^{2}-\hat{D}^{2}(B+a)\right),
$$

where we have used the symmetry property of eigenvalues of $\hat{D}$. In the lowest approximation, we omit $a_{\mu}$ in (55) and write the free energy contribution $F_{0}(B)$ similarly to the gluon contribution (42), (43) as

$$
\frac{1}{T} F_{0}^{q}(B)=-\frac{1}{2} \ln \operatorname{det}\left(m^{2}-\hat{D}^{2}(B)\right)=-\frac{1}{2} S p \int_{0}^{\infty} \zeta(t) \frac{d t}{t} e^{-t m^{2}+t \hat{D}^{2}(B)},
$$

where the sign $S p$ has the same meaning as in (43) and

$$
\hat{D}^{2}=\left(D_{\mu} \gamma_{\mu}\right)^{2}=D_{\mu}^{2}(B)+g F_{\mu \nu} \sigma_{\mu \nu} \equiv D^{2}+g \sigma F ; \quad \sigma_{\mu \nu}=\frac{1}{4 i}\left(\gamma_{\mu} \gamma_{\nu}-\gamma_{\nu} \gamma_{\mu}\right)
$$

Our aim now is to exploit the FSR to represent (56) in a form of the path integral, as it was done for gluons in (45). The equivalent form for quarks must implement the antisymmetric boundary conditions pertinent to fermions. It is easy to understand that the correct form for quark is

$$
\frac{1}{T} F_{0}^{q}(B)=-\frac{1}{2} t r \int_{0}^{\infty} \zeta(t) \frac{d t}{t} d^{4} x \overline{(D z)}_{x x}^{w} e^{-K-t m^{2}} W_{\sigma}\left(C_{n}\right)
$$

where

$$
\begin{gathered}
W_{\sigma}\left(C_{n}\right)=P_{F} P_{A} \exp \left(i g \int_{C_{n}} A_{\mu} d z_{\mu}\right) \exp g(\sigma F), \\
\overline{(D z)}_{x y}^{w}=\prod_{m=1}^{N} \frac{d^{4} \zeta(m)}{(4 \pi \varepsilon)^{2}} \sum_{n=0, \pm 1, \pm 2, \ldots}(-1)^{n} \frac{d^{4} p}{(2 \pi)^{4}} \\
\exp \left[i p\left(\sum_{m=1}^{N} \zeta(m)-(x-y)-n \beta \delta_{\mu 4}\right)\right] .
\end{gathered}
$$

One can easily check that in the case $B_{\mu}=0$ one is recovering the well known expression for the free quark gas

$$
F_{0}^{q}(\text { free quark })=-\frac{7 \pi^{2}}{180} N_{c} V_{3} T^{4} \cdot n_{f}
$$

where $n_{f}$ is the number of flavors. The derivation of (60) starting from the pathintegral form (58) is done similarly to the gluon case given in the Appendix of [8].

The loop $C_{n}$ in (58) corresponds to $n$ windings in the fourth direction. Above the deconfinement transition temperature $T_{c}$ one can visualize in (58) the appearance of the factor

$$
\Omega=\exp \left[i g \int_{0}^{\beta} B_{4}(z) d z_{4}\right] .
$$


(Note the absence of the dash sign in (61) as compared to(52) implying that in (61) $B_{4}$ is taken in fundamental representation). For the constant field $B_{4}$ and $B_{i}=0, i=1,2,3$, one obtains

$$
\langle F\rangle=-\frac{V_{3}}{\pi^{2}} \operatorname{tr}_{c} \sum_{n=1}^{\infty} \frac{\Omega^{n}+\Omega^{-n}}{n^{4}}(-1)^{n+1} .
$$

This result coincides with the obtained in the literature [27].

\section{$5 \quad$ Wilson loop and Field Correlators(FC)}

Hadron Green's functions in the form of path integrals due to FSR are expressed through Wilson loops; moreover all gluonic fields enter via these loops, and in case of quarks and gluons additional spin factors appear, namely

$$
W_{F}(C)=\operatorname{tr} P_{A} \exp i g \int_{C_{0}} A_{\mu} d z_{\mu} \cdot P_{F} \exp \int_{0}^{s} g \sum F(z(\tau)) d \tau
$$

where $\sum F=\sigma_{\mu \nu} F_{\mu \nu}$ for quarks and $2 i \hat{F}_{\mu \nu}$ for gluons, and the dash sign marks the adjoint representation for gluons in contrast to the fundamental one for quarks.

As it was shown previously in [9, 23, 28], the spin factors can be treated perturbatively in the effective Hamiltonian and this topic will be discussed in the last lecture. Here we shall consider the simplest form of Wilson loop in the arbitrary representation $D$ of the $S U(N)$,

$$
W(C)=\operatorname{tr}_{D} P_{A} \exp i g \int_{C_{0}} A_{\mu} d z_{\mu}, \quad A_{\mu}=A_{\mu}^{a} T^{a} .
$$

The nonabelian Stokes theorem [29] allows to express $W\left(C_{0}\right)$ in terms of field strength $F_{\mu \nu}$, instead of the vector potential $A_{\mu}$, this later will enable us to express the vacuum average $\left\langle W\left(C_{0}\right)\right\rangle$ through field correlators.

The easiest way to derive the Stokes theorem is via the generalized coordinate gauge [30, 31], namely one introduces the following representation for $A_{\mu}$

$$
A_{\mu}(x)=\int_{C}^{x} \frac{\partial z_{\rho}(s, x)}{\partial x_{\mu}} d z_{\nu} F_{\nu \rho}(z(s, x))
$$

Here we have introduced an arbitrary contour $C$, which is defined by a coordinate $z(s, x)$, starting at $z=x, z(1, x) \equiv x$, while for $s=0, z(0, x)=x_{0} ; x_{0}$ may also lie at infinity.

The only condition which one should impose on the contour $C(x)$ is the "automorfic condition" [31], i.e.

$$
z_{\mu}(s, x)=z_{\mu}\left(s^{\prime \prime}, z\left(s^{\prime}, x\right)\right)
$$


which means that choosing on the contour $C(x)$ some point $x^{\prime} \equiv z\left(s^{\prime}, x\right)$ to identify it with the initial point of the new contour $C\left(x^{\prime}\right)$, one gets $C\left(x^{\prime}\right)$ lying entirely on the old contour $C(x)$.

Inserting now (65) in (64) one obtains the nonabelian Stokes theorem

$$
W\left(C_{0}\right)=\operatorname{tr}_{D} P_{S} \exp i g \int_{S\left(C_{0}\right)} d \sigma_{\mu \nu}(z) F_{\mu \nu}(z)
$$

Note that the ordering $P_{S}$ in (67) is fully determined by the contour $C(x)$ in (65) and ordering of points $x$ along the contour $C_{0}$.

One can show 31] that parallel transporters $\Phi_{L}(x, y)=P \exp i g \int_{L} A_{\mu} d z_{\mu}$ in the generalized coordinate gauge are equal to unity whenever contour $L(x, y)$ lies on the contout $C(x)$. Therefore one can render the Stokes theorem (67) to the gauge-covariant form

$$
W\left(C_{0}\right)=\operatorname{tr}_{D} P_{S} \exp i g \int_{S} d \sigma_{\mu \nu}(z) F_{\mu \nu}\left(z, x_{0}\right)
$$

where

$$
F_{\mu \nu}\left(z, x_{0}\right) \equiv \Phi\left(x_{0}, z\right) F_{\mu \nu}(z) \Phi\left(z, x_{0}\right)
$$

The crucial step now is to use the cluster expansion theorem [22] and to retain only the lowest term, namely

$$
\begin{gathered}
\left\langle W\left(C_{0}\right)\right\rangle=\left\langle\operatorname{tr}_{D} P_{S} \exp i g \int_{S} d \sigma_{\mu \nu}(z) F_{\mu \nu}\left(z, x_{0}\right)\right\rangle= \\
=\operatorname{tr}_{D} \exp \left\{-\frac{g^{2}}{2} \int_{S} \int_{S} d \sigma_{\mu \nu}(z) d \sigma_{\rho \sigma}(u)\left\langle\left\langle F_{\mu \nu}\left(z, x_{0}\right) F_{\rho \sigma}\left(u, x_{0}\right)\right\rangle\right\rangle+\ldots\right\} .
\end{gathered}
$$

Here dots imply higher order terms, e.g. $\langle\langle F F F\rangle\rangle$, etc. Moreover double brackets mean cumulants, e.g. $\langle\langle A B\rangle\rangle=\langle A B\rangle-\langle A\rangle\langle B\rangle$. The angular brackets imply averaging over all gluonic fields with the standard QCD action, as in (1).

Next step is the standard representation of the bilocal field correlator in (70), made in [32]. It goes as follows. First one realizes, that when $x_{0}$ is somewhwre in the middle of surface $S$, then distances $\left|z-x_{0}\right|,\left|u-x_{0}\right|$ are of the order of $R$ - "radius" of $S$, while average distance $|z-u|$ will be of the order of the vacuum correlation length $T_{g}$.

Hence in the situation when $R \gg T_{g}$ (and this is typical situation for hadrons, since as we shall see $R \sim$ size of hadron $\sim 1 \mathrm{fm}, T_{g} \sim 0.2 \mathrm{fm}$ ) one can approximate

$$
\left\langle\left\langle F_{\mu \nu}\left(z, x_{0}\right) F_{\rho \sigma}\left(u, x_{0}\right)\right\rangle\right\rangle=\left\langle F_{\mu \nu}(z) \Phi(z, u) F_{\rho \sigma}(u) \Phi(u, z)\right\rangle+O\left(\left(\frac{T_{g}}{R}\right)^{2}\right)
$$

The lowest correlator (71) with parallel transporter connecting points $x$ and $y$ along the straight line has a general representation in terms of two scalar form-factors $D$ and $D_{1}$ [32]

$$
\frac{1}{N_{c}} \operatorname{tr}\left\langle F_{\mu \nu}(x) \Phi(x, y) F_{\lambda \sigma}(y) \Phi(y, x)\right\rangle=
$$




$$
\begin{gathered}
=\left(\delta_{\mu \lambda} \delta_{\nu \sigma}-\delta_{\mu \sigma} \delta_{\nu \lambda}\right) D(x-y)+\frac{1}{2} \partial_{\mu}\left\{\left[\left(h_{\lambda} \delta_{\nu \sigma}-h_{\sigma} \delta_{\nu \lambda}\right)+\right.\right. \\
\left.+ \text { perm }] D_{1}(x-y)\right\}, \quad h_{\mu}=x_{\mu}-y_{\mu} .
\end{gathered}
$$

It is important here that by definition the term with $D_{1}$ is a full derivative. In what follows both functions $D$ and $D_{1}$ will have a basic role in all nonperturbative dynamics in QCD, both at zero and nonzero temperature. To understand why this role is so important one must compare contribution of the bilocal FC (72) with all higher correlators, neglected in (70).

That comparison can be done at present only indirectly since the direct determination of higher FC on the lattice is difficult and still not accurate enough [33].

Happily there was another type of lattice measurement recently [34] which has allowed to estimate the contribution of higher FC with unprecendented accuracy -better then one percent!

Since the corresponding work [35] is important for understanding the structure of the QCD vacuum, we shall discuss it is some detail.

Consider static sources in representation $D$ of $S U(3)$ and define static potential between sources through the Wilson loop as follows

$$
V_{D}(R)=-\lim _{T \rightarrow \infty} \frac{1}{T} \ln \langle W(C)\rangle
$$

where the Wilson loop in the 34 -plane has the form $R \times T$ and cluster representation

$$
\langle W(C)\rangle=\operatorname{Tr}_{D} \exp \sum_{n=\eta}^{\infty} \int(i g)^{n}\langle\langle F(1) \ldots F(n)\rangle\rangle d \sigma(1) \ldots d \sigma(n) .
$$

Here we have omitted Lorentz indices and denoted

$$
F(n) d \sigma(n) \equiv F\left(x^{(n)}, x_{0}\right)=\Phi\left(x_{0}, x^{(n)}\right) E_{3}^{a}\left(x^{(n)}\right) T^{a} \Phi\left(x^{(n)}, x_{0}\right) d \sigma_{34}(x)
$$

The $S U(3)$ representations $D=3,8,6,15 a, 10,27,24,15 s$ are characterized by $3^{2}-1=8$ hermitian generators $T^{a}$ which satisfy the commutation relations $\left[T^{a}, T^{b}\right]=i f^{a b c} T^{c}$. One of the main characteristics of the representation is an eigenvalue of quadratic Casimir operator $\mathcal{C}_{D}^{(2)}$, which is defined according to $\mathcal{C}_{D}^{(2)}=T^{a} T^{a}=C_{D} \cdot \hat{1}$. Following the notations from [34] we introduce the Casimir ratio $d_{D}=C_{D} / C_{F}$, where the fundamental Casimir $C_{F}=\left(N_{c}^{2}-1\right) / 2 N_{c}$ equals to $4 / 3$ for $S U(3)$. The invariant trace is given by $\operatorname{Tr}_{D} \hat{1}=1$.

Since a simple algebra of the rank $k$ has exactly $k$ primitive Casimir-Racah operators [36] of order $m_{1}, . ., m_{k}$, it is possible to express those of higher order in terms of the primitive ones. In the case of $S U(3)$ the primitive Casimir operators are given by

$$
\mathcal{C}_{D}^{(2)}=\delta_{a b} T^{a} T^{b} ; \mathcal{C}_{D}^{(3)}=d_{a b c} T^{a} T^{b} T^{c}
$$

while the higher rank Casimir operators are defined as follows

$$
\mathcal{C}_{D}^{(r)}=d_{\left(i_{1} . i_{r}\right)}^{(r)} T^{i_{1}} . . T^{i_{r}}
$$


where the totally symmetric tensor $d_{\left(i_{1} . . i_{r}\right)}^{(r)}$ on the $S U\left(N_{c}\right)$ is expressed in terms of $\delta_{i k}$ and $d_{i j k}$ (see, for example, 37]).

The potential (73) with the definition (74) admits the following decomposition

$$
V_{D}(R)=d_{D} V^{(2)}(R)+d_{D}^{2} V^{(4)}(R)+\ldots,
$$

where the part denoted by dots contains terms, proportional to the higher powers of the quadratic Casimir as well as to higher Casimirs.

The fundamental static potential contains perturbative Coulomb part, confining linear and constant terms

$$
V_{D}(R)=\sigma_{D} R-v_{D}-\frac{e_{D}}{R}
$$

The Coulomb part is now known up to two loops [38 and is proportional to $C_{D}$. The "Casimir scaling hypothesis" [39] declares, that the confinement potential is also proportional to the first power of the quadratic Casimir $C_{D}$, i.e. all terms in the r.h.s. of $(78)$ are much smaller than the first one. In particular, for the string tensions one should get $\sigma_{D} / \sigma_{F}=d_{D}$.

This scaling law is in perfect agreement with the results found in [34, 35]. Earlier lattice calculations of static potential between sources in higher representations [39] are in general agreement with [34].

To see, why this result (to be more precise - why the impressive accuracy of the "Casimir scaling" behaviour) is nontrivial, let us examine the colour structure of a few lowest averages in the expansion (74).

The first nontrivial Gaussian cumulant in (74) is expressed through $C_{D}$ and representation-independent averages as

$$
\operatorname{Tr}_{D}\langle F(1) F(2)\rangle=\frac{C_{D}}{N_{c}^{2}-1}\left\langle F^{a}(1) F^{a}(2)\right\rangle=\frac{d_{D}}{2 N_{c}}\left\langle F^{a}(1) F^{a}(2)\right\rangle,
$$

so Gaussian approximation satisfies "Casimir scaling law" exactly. It is worth being mentioned, that this fact does not depend on the actual profile of the potential. It could happen, that the linear potential observed in [34] is just some kind of intermediate distance characteristics and changes the profile at larger $R$ (as it actually should happen in the quenched case for the representation of zero triality due to the screening of the static sources by dynamical gluons from the vacuum, or, in other words, due to gluelumps formation). The coordinate dependence of the potential is not directly related to the Casimir scaling, and can be analized at the distances which are small enough to be affected by the screening effects.

Having made these general statements, let us come back to our analysis of the contributions to the potential from different field correlators. We turn to the quartic correlator and write below several possible colour structures for it. We introduce the following abbreviation

$$
\left\langle F^{[4]}\right\rangle=\left\langle F^{a}(1) T^{a} F^{b}(2) T^{b} F^{c}(3) T^{c} F^{d}(4) T^{d}\right\rangle=T^{a} T^{b} T^{c} T^{d}\left\langle F^{[4]}\right\rangle^{a b c d}
$$


where the Lorentz indices and coordinate dependence are omitted for simplicity of notation. One then gets, with some work in the last case the following possible structures

$$
\begin{aligned}
& \left\langle F^{[4]}\right\rangle^{a b c d} \sim \delta_{a b} \delta_{c d} \quad\left\langle F^{[4]}\right\rangle \sim C_{D}^{2} \cdot \hat{1} \\
& \left\langle F^{[4]}\right\rangle^{a b c d} \sim \delta_{a c} \delta_{b d} \quad\left\langle F^{[4]}\right\rangle \sim\left(C_{D}^{2}-\frac{1}{2} N_{c} C_{D}\right) \cdot \hat{1} \\
& \left\langle F^{[4]}\right\rangle^{a b c d} \sim f_{\text {ade }} f_{\text {cbe }} \quad\left\langle F^{[4]}\right\rangle \sim-\frac{1}{4} N_{c}^{2} C_{D} \cdot \hat{1} \\
& \left\langle F^{[4]}\right\rangle^{a b c d} \sim f_{\text {ace }} f_{\text {bde }} \quad\left\langle F^{[4]}\right\rangle=0 \\
& \left\langle F^{[4]}\right\rangle^{a b c d} \sim f_{\text {apm }} f_{\text {bpn }} f_{\text {dem }} f_{\text {cen }} \quad\left\langle F^{[4]}\right\rangle \sim \frac{9}{4}\left(C_{D}^{2}+\frac{1}{2} C_{D}\right) \cdot \hat{1}
\end{aligned}
$$

The operator $\mathcal{C}_{D}^{(3)}$ enters together with $\mathcal{C}_{D}^{(2)}$ at higher orders. Notice, that the terms, proportional to the square of $C_{D}$ appear in both the $\delta \delta$ parts (the first and the second strings) and higher order interaction parts (the last string). Mnemonically the $C_{D}$ - proportional components arise from the diagrams where the noncompensated colour flows inside while the $C_{D}^{2}$ - components describe the interaction of two white objects. It is seen, that the Casimir scaling does not mean "quasifree gluons", instead it means roughly speaking "quasifree white multipoles" (see discussion at the end of the paper).

Let us analyse the data from [34 quantitatively. We have already mentioned, that the Coulomb potential between static sources is proportional to $C_{D}$ up to the second loop (and possibly to all orders, this point calls for further study) and hence we expect contributions proportional to $C_{D}^{2} \sim d_{D}^{2}$ to the constant and linear terms, i.e. we rewrite $(79)$ as follows

$$
V_{D}(R)=d_{D} V^{(2)}(R)+d_{D}^{2}\left(v_{D}^{(4)}+\sigma_{D}^{(4)} R\right) .
$$

and all higher contributions are omitted. Here $v_{D}^{(4)}, \sigma_{D}^{(4)}$ measure the $d_{D}^{2}$-contribution of the cumulants higher than Gaussian to the constant term and string tension respectively. The results of the fitting of the data from [34 with (81) for some representations are shown in the Table 1.

All numbers in the Table 1 are dimensionless and given in lattice units. The author of [34] used anisotropic lattice with the spatial unit $a_{s}^{-1}=2.4 \mathrm{GeV}$.

Several comments are in order. First of all it is seen that the Casimir scaling behaviour holds with very good accuracy, better than $1 \%$ in all cases in the table 1 with the reasonable $\chi^{2}$. It should be stressed, that any possible systematic errors which could be present in the procedure used in [34] must either obey the Casimir scaling too or be very tiny, otherwise it would be unnatural to have the matching with such high precision.1 Nevertheless, the terms violating the scaling are also clearly seen. While the value of the constant term $v_{D}^{(4)}$ is found to be compatible with zero within the error bars, it is not the case for $\sigma_{D}^{(4)}$. We have not found any

\footnotetext{
${ }^{1}$ Since we are mostly interested in the relative quantities, their actual magnitude in the physical units is of no prime importance for us. This is another reason why we do not discuss possible systematical errors of [34] and finite volume effects.
} 
Table 1: The Casimir-scaling and Casimir-violating string tensions and shifts. Based on the data from G.Bali, hep-lat/9908021. [34]

\begin{tabular}{|c|c|c|c|c|c|c|}
\hline$D$ & $\sigma_{D}^{(4)} \cdot 10^{4}$ & $\Delta \sigma_{D}^{(4)} \cdot 10^{4}$ & $v_{D}^{(4)} \cdot 10^{4}$ & $\Delta v_{D}^{(4)} \cdot 10^{4}$ & $\left|\sigma_{D}^{(4)} / \sigma_{D}^{(2)}\right|$ & $\chi^{2} / N$ \\
\hline 8 & -3.486 & 1.2 & -2.513 & 2.8 & 0.004 & $19.22 / 43$ \\
\hline 6 & -6.428 & 1.2 & 0.950 & 2.6 & 0.007 & $25.76 / 42$ \\
\hline $15 \mathrm{a}$ & -5.244 & 0.55 & -0.5611 & 1.1 & 0.003 & $39.06 / 42$ \\
\hline 10 & -4.925 & 0.50 & 0.2489 & 1.0 & 0.003 & $22.05 / 41$ \\
\hline
\end{tabular}

sharp dependence of $\sigma_{D}^{(4)}$ on the representation $D$, which confirms the validity of the expansion (81) and shows, that the omitted higher terms do not have significant effect in this case. Notice the negative sign of the string tension correction. In euclidean metric it trivially follows from the fact, that the fourth order contribution is proportional to $(i g)^{4}>0$ while the Gaussian term is multiplied by $(i g)^{2}<0$ for real $g$.

The analysis given above allows to draw important conclusions about the QCD vacuum:

1) The contribution of higher FC to the static potential is less than $1 \%$ at all measured distances $0.1 \mathrm{fm} \leq R \leq 1.3 \mathrm{fm}$. (Another possible scenario is that all higher FC are fine-tuned to cancel exactly all terms proportional to $C_{D}^{n}, n>1$; but this fine-tuning seems rather artificial).

2) The lowest correlators $D$ and $D_{1}$ should describe dynamics of the Wilson loop (i.e. the dynamics of nonperturbative QCD with accuracy better than $1 \%$. This fact can be checked in calculations of physical amplitudes, e.g. we shall give exmples of our predictions in the last lecture.

3) All modes of the QCD vacuum which are based on classical solutions are strongly limited by the observed Casimir scaling, since a classical lump (e.g. an instanton or a dyon) generate a big Quartic (and higher) FC.

E.g. the density of instanton gas (in the $\mathrm{SU}(2)$ case) in the QCD vacuum has an upper bound of $\frac{N}{V}=0.2 \mathrm{fm}^{-4}$ (and even smaller if one considers smaller distances). This should be compared with the normal density of instanton gas of $1 \mathrm{fm}^{-4}$ pro- 
viding the standard gluonic condensate.

The picture of the QCD vacuum which emerges from the Casimir scaling is the picture of stochastic ensemble of small white dipoles, where only any two adjacent points are correlated, while all the rest are statistically independent. This picture is ideal for description in terms of lowest field correlators, $D(x)$ and $D_{1}(x)$ at all temperatures, and we shall indeed show how easily one can describe dynamics of different precesses in QCD.

4) As a by-product the results of $[34,35]$ give a strong upper bound on the socalled Luescher-Weissz term in the potential [40], $V_{L W}=-\frac{\pi}{12 R}$, since the latter violates Casimir scaling. This means that the QCD string is either not the NambuGoto string, used in [40, or else one should use properly the finite lattice size for vibrating strings - this is an interesting problem for the future.

\section{$6 \quad$ Field correlators at zero and nonzero tempera- ture}

Since $D(x)$ and $D_{1}(x)$ are basic elements of the nonperturbative QCD dynamics, it is important to find them as functions of distance $x$ and use later on as an input.

The only standard way to get information on $D, D_{1}$ before was the lattice simulation method, and lattice measurements of $D, D_{1}$ have been done both at $T=0$ [41] at $T>0$ [42.

At the end of these lectures we shall describe other methods of calculating field correlators and in particular $D$ and $D_{1}$, but now we shall describe in detail results of the Pisa group.

For $T=0$ these data are presented in Fig.1, and can be parametrized by the form

$$
\begin{gathered}
D(x)=A e^{\mu x}+\frac{a e^{-\mu_{1} x}}{x^{4}} \\
D_{1}(x)=B e^{\mu^{\prime} x}+\frac{b e^{-\mu_{1}^{\prime} x}}{x^{4}}
\end{gathered}
$$

The last terms in (82), (83) proportional to $x^{-4}$ are clearly of perturbative origin, moderated at large distances by screening factors. The most important information is contained in the first terms in (82), (83) proportional to exponents. It is striking that numerically $\mu=\mu^{\prime} \simeq 1 \mathrm{GeV}$ for the unquenched case.

At the end of the lectures we shall explain the appearance of exponents in (82). (83) and the mass values around $1 \mathrm{GeV}$ - this will be connected to the masses of the so-called gluelumps - bound states of gluons with a static adjoint source. These masses have been calculated recently both on the lattice [43] and analytically [44].

Physically appearance of large masses - of the order of $1 \mathrm{GeV}$ leads to very important consequences: 
i) First, the gluon correlation length $T_{g} \equiv 1 / \mu \approx 0.2 \mathrm{fm}$ is small as compared to the generic hadronic size and implies that stochastic picture of small independent white dipoles which was discussed in previous section.

ii) As a consequence of small $T_{g}$ one can develop the potential picture of hadrons (see [23, 28] for more discussion), with quarks and valence gluons interacting via a string instantaneously without retardation, while nonpotential corrections are proportional to some powers of $\left(T_{g} / R_{h}\right)$, where $R_{h}$ is the typical hadron size.

iii) Finally, smallness of $T_{g}$ puts serious limitation on the use of some standard methods in QCD. E.g. the application of QCD sum rules strictly speaking implies large values of $T_{g}$, namely $T_{g} \gg R_{h}$, otherwise the notion of gluon condensate and its derivatives loses its meaning.

The same is true for the Voloshin-Leutwyler method, based on the constant condensate contribution to the heavy quarkonia dynamics. One can estimate that the inequality $R_{Q \bar{Q}} \leq T_{g}$ holds true for toponium and hardly for bottomonium.

Knowing $D$ and $D_{1}$ one can calculate all the dynamics of heavy quarkonia (and also of other hadrons - in the next sections).

In particular the string tension is defined by the nonperturbative part of $D(x)$ [32]

$$
\sigma=\frac{1}{2} \int d^{2} x D(x)
$$

Note that $D_{1}$ does not contribute, being the full derivative, and the perturbative part of $D(x)$ is cancelled by the corresponding perturbative parts of triple and higher correlators [45]. The whole static potential, as expressed through $D$ and $D_{1}$ has the form

$$
V(R)=2 R \int_{0}^{R} d \lambda \int_{0}^{\infty} d \nu D(\lambda, \nu)+\int_{0}^{R} \lambda d \lambda \int_{0}^{\infty} d \nu\left[-2 D(\lambda, \nu)+D_{1}(\lambda, \nu)\right]
$$

Here $D(\lambda, \nu)=D\left(\sqrt{\lambda^{2}+\nu^{2}}\right)$ because of Euclidean $O(4)$ invariance. Note that the first term on the r.h.s. of (85) yields at large $R$ the term $\sigma R$, while the second term adds there a constant. The perturbative contribution to $V(R)$ can be included in $D_{1}$, or considered separately, in both cases one obtains the form

$$
V(R)=\sigma R-v-4 / 3 \frac{\alpha_{s}(R)}{R}
$$

observed many times on the lattice and recently in [34].

We shall comment in what follows on the NP dependence of $\alpha_{s}(R)$, but now we turn to spin-dependent corrections to the potential, calculated in [28, 46], i.e. spin-orbit potentials $V_{1}, V_{2}$, tensor force $V_{3}$ and spin-spin potential $V_{4}$ (we exploit the standard notations of Eichten and Feinberg [47]). All these potentials are expressed through $D$ and $D_{1}$ only and in this way the dynamics of heavy quarkonia is fully determined.

The corresponding calculations have been done repeatedly and successfully during last 10 years in 48-51. In the Tables 2 and 3, given are the known levels of 
charmonium and bottomonium calculated using the lattice data on $D, D_{1}$ from in a standard way. One can see a very good description of all levels, which far exceeds in quality the newly developed NRQCD methods.

In the Table 2 listed are bottomonium level differences, which have more physical significance than the absolute values of levels, since the heavy quark mass is not known and used as an input parameter. Calculations in 51 have been done both in relativistic and nonrelativistic kinematics, and one can estimate therefore the accuracy of $1 / \mathrm{M}$ expansion usually done in the framework of NRQCD. One can see that corrections can be very significant, especially for $P$-wave levels, where the matrix element of the $1 / r^{3}$ operator is increased up to $20 \%$ in relativistic calculations [51].

Similar calculations have been done for charmonium in [50], again with very good results. An interesting comparison of these and similar results is contained in [52], where the Voloshin-Leutwyler method was used, and shown to be applicable (if any) only to ground state of bottomonium.

Table 2: Bottomonium level differences $(\mathrm{MeV})$ for the Schrödinger and Salpeter equations from [5]

\begin{tabular}{|c|c|c|c|c|c|}
\hline Masses differences & \multicolumn{2}{|c|}{$\begin{array}{c}\text { Set I, } \alpha_{\text {eff }}=0.3545 \\
m=4.737 \mathrm{GeV}\end{array}$} & \multicolumn{2}{c|}{$\begin{array}{c}\text { Set II, } \alpha_{\text {eff }}=0.36 \\
m=4.81 \mathrm{GeV}\end{array}$} & $\begin{array}{c}\text { Exp.val } \\
(\mathrm{MeV})\end{array}$ \\
& \multicolumn{2}{|c|}{$\sigma=0.20 \mathrm{GeV}{ }^{2}$} & \multicolumn{2}{c|}{$\sigma=0.18 \mathrm{GeV}^{2}$} & \\
\hline & Rel. & Nonrel. & Rel. & Nonrel. & \\
\hline$M(2 S)-M(1 S)$ & 554.34 & 551.97 & 556.55 & 550.03 & $562.9 \pm 0.5$ \\
$M(3 S)-M(2 S)$ & 350.43 & 354.78 & 335.62 & 338.49 & $332.0 \pm 0.8$ \\
$M(4 S)-M(3 S)\left({ }^{a}\right)$ & 285.93 & 291.83 & 270.63 & 275.30 & $224.7 \pm 4.0$ \\
$M(1 P)-M(1 S)$ & 458.04 & 439.66 & 473.49 & 450.15 & $439.8 \pm 0.9$ \\
$M(2 P)-M(1 P)$ & 359.67 & 366.75 & 342.55 & 348.70 & $359.8 \pm 1.2$ \\
$M(2 S)-M(1 P)$ & 96.31 & 112.31 & 83.07 & 99.88 & $123.1 \pm 1.0$ \\
$M(3 S)-M(2 P)$ & 87.06 & 100.34 & 72.82 & 89.67 & $95.3 \pm 1.0$ \\
\hline
\end{tabular}

$\left.{ }^{a}\right)$ The $4 S$ level lies above the $b \bar{B}$ threshold and the corresponding threshold correction was not taken into account, which explains the discrepancy. 
Table 3: The spin-averaged mass level differences in charmonium ${ }^{a)}$ (in $\mathrm{MeV})$

\begin{tabular}{|c|c|c|c|}
\hline & $\begin{array}{c}\text { Experimental } \\
\text { values }^{b)} \\
(\mathrm{MeV})\end{array}$ & $\begin{array}{c}\text { Set } C \\
M=1.48 \mathrm{GeV} \\
\sigma=0.18 \mathrm{GeV}^{2} \\
\alpha_{s}\left(\mu_{0}\right)=0.365 \\
\mu_{0}=0.909 \mathrm{GeV}, \\
\tilde{\alpha}_{V}=0.42\end{array}$ & $\begin{array}{c}\text { Set } D \\
m=1.40 \mathrm{GeV}, \\
\sigma=0.183 \mathrm{GeV}^{2}, \\
\alpha_{s}\left(\mu_{0}\right)=0.312, \\
\mu_{0}=1.357 \mathrm{GeV}, \\
\tilde{\alpha}_{V}=0.39\end{array}$ \\
\hline$M(2 S)-M(1 S)$ & $595.39 \pm 1.91$ & 591.30 & 586.09 \\
\hline$M(1 P)-M(1 S)$ & $457.92 \pm 1.0$ & 460.68 & 445.93 \\
\hline$M(2 P)-M(1 P)$ & $137.47 \pm 1.77$ & 130.62 & 140.15 \\
\hline
\end{tabular}

a) all matrix elements defining spin effects were calculated for spinless Salpeter equation.

b) $M(n L)$ means the spin-averaged mass.

\section{The Field Correlator Method for light quarks}

Let us start with the meson Green's function and use for that FSR for both quark and antiquark.

$$
G_{M}(x, y)=\left\langle\operatorname{tr} \Gamma^{(f)}(m-\hat{D}) \int_{0}^{\infty} d s \int_{0}^{\infty} d \bar{s} e^{-K-\bar{K}}(D z)_{x y}(D \bar{z})_{x y} \Gamma^{(i)}(\bar{m}-\hat{\bar{D}}) W_{F}\right\rangle
$$

Here the bar sign refers to the antiquark, and

$$
W_{F}=P_{A} P_{F} \exp \left(i g \oint d z_{\mu} A_{\mu}\right) \exp \left(g \int_{0}^{s} d \tau \sigma_{\mu \nu}^{(1)} F_{\mu \nu}\right) \exp \left(-g \int_{0}^{\bar{s}} \sigma_{\mu \nu}^{(2)} F_{\mu \nu} d \bar{\tau}\right)
$$

In (87) enter integrations over proper times $s, \bar{s}$ and $\tau, \bar{\tau}$, which also play the role of ordering parameter along the trajectory, $z_{\mu}=z_{\mu}(\tau), \quad \bar{z}_{\mu}=\bar{z}_{\mu}(\bar{\tau})$.

It is convenient to go over to the actual time $t \equiv z_{4}$ of the quark (or antiquark), defining the new quantity $\mu(t)$, which will play very important role in what follows

$$
2 \mu(t)=\frac{d t}{d \tau}, \quad t \equiv z_{4}(\tau)
$$

For each quark (or antiquark and gluon) one can rewrite the path integral (34), (87) as follows

$$
\int_{0}^{\infty} d s\left(D^{4} z\right)_{x y \cdots}=\text { const } \int D \mu(t)\left(D^{3} z\right)_{x y \cdots}
$$


where $\left(D^{3} z\right)_{x y}$ has the same form as in (26) but with all 4-vectors replaced by 3vectors, and the path integral $D \mu(t)$ is supplied with the proper integration measure, which is derived from the free motion Lagrangian.

In general $\mu(t)$ can be a strongly oscillating function of $t$ due to the Zitterbewegung. In what follows we shall use the steepest descent method for the evaluation of the integral over $D \mu(t)$, with the extremal $\mu_{0}(t)$ playing the role of effective or constituent quark mass. We shall see that in all cases, where spin terms can be considered as a small perturbation, i.e. for majority of mesons, $\mu_{0}$ is positive and rather large even for vanishing quark current masses $m, \bar{m}$, and the role of Zitterbewegung is small (less than $10 \%$ from the comparison to the light-cone Hamiltonian eigenvalues, see [53, 54 for details).

Now the kinetic terms can be rewritten using (89) as

$$
K+\bar{K}=\int_{0}^{T} d t\left\{\frac{m^{2}}{2 \mu(t)}+\frac{\mu(t)}{2}\left[\left(\dot{z}_{i}(t)\right)^{2}+1\right]+\frac{\bar{m}^{2}}{2 \bar{\mu}(t)}+\frac{\bar{\mu}(t)}{2}\left[\left(\dot{\bar{z}}_{i}(t)\right)^{2}+1\right]\right\}
$$

where $T=x_{4}-y_{4}$. In the spin-dependent factors the corresponding changes are

$$
\int_{0}^{s} d \tau \sigma_{\mu \nu} F_{\mu \nu}=\int_{0}^{T} \frac{d t}{2 \mu(t)} \sigma_{\mu \nu} F_{\mu \nu}(z(t))
$$

In what follows in this section we shall systematically do perturbation expansion of the spin terms, which contribute to the total mass corrections of the order of 10$15 \%$ for lowest mesons and much smaller for high excited states. This perturbative approach fails however for pions (and kaons) where chiral degrees of freedom should be taken into account. In this case other equation should be considered [55, 56]. Therefore as the starting approximation we shall use the Green's functions of mesons made of spinless quarks, which amounts to neglect in (87), (88) terms $(m-\hat{D}),(\bar{m}-$ $\hat{\bar{D}})$ and $\sigma_{\mu \nu} F_{\mu \nu}$. As a result one has

$$
G_{M}^{(0)}(x, y)=\mathrm{const} \int D \mu(t) D \bar{\mu}(t)\left(D^{3} z\right)_{x y}\left(D^{3} \bar{z}\right)_{x y} e^{-K-\bar{K}}\langle W\rangle .
$$

Our next approximation is the neglect of perturbative exchanges in $\langle W\rangle$, (they will be restored in the final expression for Hamiltonian) which yields for large Wilson loops, $R, T \gg T_{g}$,

$$
\langle W\rangle_{B}=\text { const } \exp \left(-\sigma S_{\min }\right)
$$

where $S_{\min }$ is the minimal area inside the given trajectories of quark and antiquark,

$$
S_{\min }=\int_{0}^{T} d t \int_{0}^{1} d \beta \sqrt{\operatorname{det} g}, \quad g_{a b}=\partial_{a} w_{\mu} \partial_{b} w^{\mu}, a, b=t, \beta .
$$

The Nambu-Goto form of $S_{\text {min }}$ cannot be quantized due to the square root and one has to use the auxiliary field approach [57] with functions $\nu(\beta, t)$ and $\eta(\beta, t)$ to 
get rid of the square root, as it is usual in string theories. As the result the total Euclidean action becomes 58

$$
\begin{aligned}
A=K+ & \bar{K}+\sigma S_{\min }=\int_{0}^{T} d t \int_{0}^{1} d \beta\left\{\frac{1}{2}\left(\frac{m^{2}}{\mu(t)}+\frac{\bar{m}^{2}}{\bar{\mu}(t)}\right)+\frac{\mu_{+}(t)}{2} \dot{R}^{2}+\right. \\
+ & \left.\frac{\tilde{\mu}(t)}{2} \dot{r}^{2}+\frac{\nu}{2}\left[\dot{w}^{2}+\left(\frac{\sigma}{\nu}\right)^{2} r^{2}-2 \eta(\dot{w} r)+\eta^{2} r^{2}\right]\right\} .
\end{aligned}
$$

Here $\mu_{+}=\mu+\bar{\mu}, \quad \tilde{\mu}=\frac{\mu \bar{\mu}}{\mu+\bar{\mu}}, \quad R_{i}=\frac{\mu z_{i}+\bar{\mu} \bar{z}_{i}}{\mu+\bar{\mu}}, \quad r_{i}=z_{i}-\bar{z}_{i}$. Note, that integrations over $\mu, \nu$ and $\eta$ effectively amount to the replacement by their extremum values [58].

Performing Gaussian integrations over $R_{\mu}$ and $\eta$ one arrives in the standard way at the Hamiltonian (we take $m=\bar{m}$ for simplicity)

$$
\begin{gathered}
H=\frac{p_{r}^{2}+m^{2}}{\mu(\tau)}+\mu(\tau)+\frac{\hat{L}^{2} / r^{2}}{\mu+2 \int_{0}^{1}\left(\beta-\frac{1}{2}\right)^{2} \nu(\beta) d \beta}+ \\
\quad+\frac{\sigma^{2} r^{2}}{2} \int_{0}^{1} \frac{d \beta}{\nu(\beta)}+\int_{0}^{1} \frac{\nu(\beta)}{2} d \beta,
\end{gathered}
$$

where $p_{r}^{2}=(\mathbf{p r})^{2} / r^{2}$, and $L$ is the angular momentum, $\hat{L}=(\mathbf{r} \times \mathbf{p})$.

The physical meaning of the terms $\mu(t)$ and $\nu(\beta)$ can be understood when one finds their extremal values. E.g. when $\sigma=0$ and $L=0$, one finds from (97)

$$
H_{0}=2 \sqrt{\mathbf{p}^{2}+m^{2}}, \quad \mu_{0}=\sqrt{\mathbf{p}^{2}+m^{2}}
$$

so that $\mu_{0}$ is the energy of the quark. Similarly in the limiting case $L \rightarrow \infty$ the extremum over $\nu(\beta)$ yields:

$$
\nu_{0}(\beta)=\frac{\sigma r}{\sqrt{1-4 y^{2}\left(\beta-\frac{1}{2}\right)^{2}}}, \quad H_{0}^{2}=2 \pi \sigma \sqrt{L(L+1)}
$$

so that $\nu_{0}$ is the energy density along the string with the $\beta$ playing the role of the coordinate along the string.

Let us start with the $L=0$ case. Taking extremum in $\nu(\beta)$ one has

$$
H_{1}=\frac{p_{r}^{2}+m^{2}}{\mu(t)}+\mu(t)+\sigma r .
$$

Here $\mu(t)$ is to be found also from the extremum amd is therefore an operator in Hamiltonian formalism. Taking extremum in $\mu(t)$ one obtains

$$
H_{2}=2 \sqrt{p_{r}^{2}+m^{2}}+\sigma r .
$$

The Hamiltonian (101) is what one traditionally exploits in the relativistic quark model (RQM) [59 (apart from color Coulomb and spin-dependent terms to be discussed below). The RQM was an essential step in our understanding of hadronic 
spectra. At the same time the usual input of RQM contains too many parameters and the model was introduced rather ad hoc. Another deficiency of this model at this point is twofold:

i) one usually takes $m$ in (98),(101) to be constituent quark mass of the order of 100-200 MeV, which is introduced as an input. Instead we have in (97),(99),(101) the current quark mass renormalized at the reasonable scale of $1 \mathrm{GeV}$. Hence it is almost zero for light quarks;

ii) the form (101) is used in RQM for any $L$, and to this end one writes in (101) $p_{r}^{2} \rightarrow \mathbf{p}^{2}$.

However the Regge slope for both (100) and (101) is $1 / 8 \sigma$ instead of the string slope $\frac{1}{2 \pi \sigma}$, which occurs for the total Hamiltonian (97), since the RQM Hamiltonian (101) does not take into account string rotation.

Still for $L=0$ the form (101) is a good starting approximation, and it is rewarding that our systematic approach makes here contact with RQM. Sometimes it is convenient to use instead of (101) a more tractable form (100) where $\mu(t)=\mu_{0}$ and $\mu_{0}$ is to be found from the extremum of the eigenvalue of Hamiltonian $H_{1}$ :

$$
H_{1} \psi=M_{1} \psi, \quad M_{1}=M_{1}\left(\mu_{0}\right), \quad \frac{\partial M_{1}}{\partial \mu_{0}}=0
$$

Equation (100), (101) can be reduced to the Airy equation with the eigenvalues written as (for $m=0$ )

$$
M_{n}^{(1)}=4 \mu_{0}(n), \quad \mu_{0}(n)=\sqrt{\sigma}\left(\frac{a(n)}{3}\right)^{3 / 4}
$$

where $a(n)$ is the corresponding zero of the Airy function. The first comprehensive study of the equation (102) with fixed $\mu(t) \equiv m$ was done in [60], where also $a(n)$ are quoted.

It is easy to apply the Hamiltonian (97) to the case of light, [23, 60, 61] heavy [48][51] and heavy-light mesons [24, 62]. We refer here to [61] where these applications and also hybrid calculations have been successfully done. Again, the most important point of the described formalism is the natural occurence of the constituent mass of quark and gluon, which is calculated through the string tension (and $\alpha_{s}$ ) in the unique way, as shown in Eq.(102).

Since this point is very important from both phenomenological and physical point of view, we shall discuss here in more detail the case of baryon magnetic moment (defined through quark constituent mass) and spectrum of glueballs (where constituent mass of gluons enter in a decisive way).

For baryons the Hamiltonian is derived from the Green's function of a baryon in the same way as it was done above for mesons.

We refer the reader to [63]-65] for details of derivation. The result is

$$
H=\sum_{k=1}^{3}\left(\frac{m_{k}^{2}}{2 \mu_{k}}+\frac{\mu_{k}}{2}\right)+\left(-\frac{\partial^{2}}{\partial \boldsymbol{\xi}^{2}}-\frac{\partial^{2}}{\partial \boldsymbol{\eta}^{2}}\right)+\sigma \sum_{k=1}^{3}\left|\mathbf{r}^{(k)}\right| .
$$


Here $m_{k}$ are current quark masses defined at the scale of $1 \mathrm{GeV}, \mu_{k}$ - the corresponding constituent masses (energies) to be calculated in what follows, $\mathbf{r}^{(k)}$ is the vector from position of the $k$-th quark to the string junction. The coordinates $\boldsymbol{\xi}, \boldsymbol{\eta}$ are Jacobi combinations defined through $\mathbf{r}^{(k)}$ in a standard way.

The eigenvalues of (104) $M_{n}(\mu)$ can be found in a simple way with good accuracy using the the hyper-spherical explansion method (menthod of the $K$ - harmonics) [66]. Assuming equal masses $M_{k}=m$ and hence equal constituent masses $\mu_{k}=\mu$, one obtains equation for the definition of $\mu$

$$
\left.\frac{\partial M_{n}(\mu)}{\partial \mu}\right|_{\mu=\mu^{(0)}}=0 \text {. }
$$

¿From that we find $\mu \equiv \mu_{u}$ for baryon consisting of 3 light quarks

$$
\mu_{u}=2 \sqrt{\frac{2 \sigma}{\pi}}\left[\frac{2}{3 \cdot 5^{1 / 3}}\left(1+\frac{2}{3 \sqrt{5}}\right)\right]^{3 / 4}=0.957 \sqrt{\sigma} .
$$

Choosing $\sigma=0.15 \mathrm{GeV}^{2}$ (as it accepted for baryons phenomenologically 67 and recently found microscopically 68) one has $\mu_{u}=0.37 \mathrm{GeV}$. For the strange quarks, $m_{s}=0.175 \mathrm{GeV}$, one instead has $\mu_{s}=0.46 \mathrm{GeV}$.

Now comes the most dramatic moment, since $\mu_{u}, \mu_{s}$ are directly connected to the baryon magnetic moment. Namely, similarly to the color-magnetic term, also interaction with usual magnetic field has the form

$$
\delta A=\sum_{k=1}^{3} \int_{0}^{s_{k}} d \tau_{k} e_{k} \boldsymbol{\sigma}^{(k)} \mathbf{B}=\sum_{k=1}^{3} \int_{0}^{T} \frac{e_{k} \boldsymbol{\sigma}^{(k)} \mathbf{B}}{2 \mu_{k}} d t,
$$

where $e_{k}$ is the electric charge of the quark, $\boldsymbol{\sigma}^{(k)}$ is the corresponding spin operator, and the definition (89) of the constituent mass was used.

Introducing the $z$-component of the magnetic moment operator

$$
\hat{\mu}_{z}=\sum_{k=1}^{3} \frac{e_{k} \sigma_{z}^{(k)}}{2 \mu_{k}}
$$

one can write the BMM as matrix elements

$$
\mu_{B} \equiv\left\langle\Psi_{B}\left|\hat{\mu}_{z}\right| \Psi_{B}\right\rangle
$$

where $\Psi_{B}$ is the eigenfunction of (104), and $\mu_{k}$ is taken at the stationary point, given by (105).

For the baryon wave function we shall take here the simplest approximation, namely

$$
\Psi_{B}=\Psi^{\text {symm }}(r) \psi^{\text {symm }}(\sigma, f) \psi^{a}(\text { color }),
$$

where $\psi(\sigma, f)$ is the spin-flavour part of the wave function. 
Table 4: Magnetic moments of baryons (in nuclear magnetons) computed using Eqs.(11),(108),(14) in comparison with experimental data from PDG [24]

\begin{tabular}{|l|l|l|l|l|l|l|l|l|l|l|}
\hline Baryon & $p$ & $n$ & $\Lambda$ & $\Sigma^{-}$ & $\Sigma^{0}$ & $\Sigma^{+}$ & $\Xi^{-}$ & $\Xi^{0}$ & $\Omega^{-}$ & $\Delta^{++}$ \\
\hline Present work & 2.54 & -1.69 & -0.69 & -0.90 & 0.80 & 2.48 & -0.63 & -1.49 & -2.04 & 436 \\
\hline Experiment & 2.79 & -1.91 & -0.61 & -1.16 & & 2.46 & -0.65 & -1.25 & -2.02 & 4.52 \\
\hline
\end{tabular}

Results of calculations of baryon magnetic moments [65] are given in Table 4 in nuclear magnetons.

Note that all our predicted values of $\mu^{(B)}$ are calculated only through the string tension and this is the first calculation of this sort, which tells that the string picture of baryons (we call it the QCD string model) is very good starting approximation - agreement of the calculations with experiment is on average better than of any other approach.

One more example in this section will illustrate the universality of the approach, where the notion of constituent gluon mass enters and this quantity is explicitly calculated.

The example is the glueball spectrum, calculated in this approach in [69, 70].

Since gluons have no current masses, $m_{k}=0$, the hamiltonian for angular momentum $L=0$ has the form (without spin and $L$ corrections)

$$
H_{0}=\frac{\mathbf{p}^{2}}{\mu_{0}}+\mu_{0}+\sigma_{a d j} r
$$

Here $\sigma_{a d j}=\frac{9}{4} \sigma_{\text {fund }}, \quad \sigma_{\text {fund }}=0.18 \mathrm{GeV}^{2}$. The eigenvalues are easily obtained from (103),

$$
M_{0}(n)=4 \mu_{0}(n), \quad \mu_{0}(n)=\sqrt{\sigma_{a d j}}\left(\frac{a(n)}{3}\right)^{3 / 4},
$$

and $a(L=0, n=0)=2.338, \quad a(L=1, n=0)=3.36, \quad a(L=0, n=1)=4.088$.

To this Hamiltonian $H_{0}$ one should add spin-dependent terms $H_{s}$ (totally expressed through $\alpha_{s}$ and $D, D_{1}$ see 61 andrefs.therein) and orbital correction $H_{L}$ derived in [58]. Referring the reader to [69, 70] for details here we only give the Table of galueball masses in comparison with two sets of lattice calculations (for the same value of $\sigma_{f}=0.238 \mathrm{GeV}^{2}$, chosen on the lattice).

One can notice a remarkable agreement between analytic and lattice data. Note also that both calculations have essentially the same input: the string tension $\sigma_{f}$, while spin splittings are also defined by the value of the strong coupling $\alpha_{s}$. It means that the Field Correlator Method is also successful in describing gluon bound system and the notion of valence gluon and its constituent mass (energy) is fully supported by independent calculations.

Let us turn now to another gluon system, which has important theoretical rather than experimental meaning. This is the gluelump system calculated both on the 
Table 5: Comparison of predicted glueball masses with lattice data (for $\sigma_{f}=0.238$ $\mathrm{GeV}^{2}$ )

\begin{tabular}{|l|l|l|l|}
\hline$J^{P C}$ & $M(\mathrm{GeV})$ & \multicolumn{2}{|c|}{ Lattice data } \\
\cline { 3 - 4 } & This work & Ref. [1] & Ref. [3] \\
\hline $0^{++}$ & 1.58 & $1.73 \pm 0.13$ & $1.74 \pm 0.05$ \\
$0^{++*}$ & 2.71 & $2.67 \pm 0.31$ & $3.14 \pm 0.10$ \\
$2^{++}$ & 2.59 & $2.40 \pm 0.15$ & $2.47 \pm 0.08$ \\
$2^{++*}$ & 3.73 & $3.29 \pm 0.16$ & $3.21 \pm 0.35$ \\
$0^{-+}$ & 2.56 & $2.59 \pm 0.17$ & $2.37 \pm 0.27$ \\
$0^{-+*}$ & 3.77 & $3.64 \pm 0.24$ & \\
$2^{-+}$ & 3.03 & $3.1 \pm 0.18$ & $3.37 \pm 0.31$ \\
$2^{-+*}$ & 4.15 & $3.89 \pm 0.23$ & \\
$3^{++}$ & 3.58 & $3.69 \pm 0.22$ & $4.3 \pm 0.34$ \\
$1^{--}$ & 3.49 & $3.85 \pm 0.24$ & \\
$2^{--}$ & 3.71 & $3.93 \pm 0.23$ & \\
$3^{--}$ & 4.03 & $4.13 \pm 0.29$ & \\
\hline
\end{tabular}

lattice [43] and in the framework of the present method in 44. There is a remarkable agreement between the states defined in the same way (through space-like links).

The most important feature of gluelumps is that the lowest masses with electric quantum numbers $1^{--}$correspond exactly to the masses observed in the exponents by the Pisa group 41. And indeed calculations in 44 for the electric gluelump (which is described by electric correlator $\left\langle E_{i} \Phi E_{k}\right\rangle$ ) yield for the mass $\mu \approx 1 \mathrm{GeV}$ in agreement with [41]. Note however that magnetic gluelumps with quantum numbers $1^{+-}$, probably could not been observed in [41], since quantum numbers were not specifically projected in [41], and therefore presumably only lowest mass could be observed.

Analytic calculation of gluelumps, i.e. of field correlators with given given quantum numbers, signifies a new important period in the development of the Method as whole: the functions $D(x)$ and $D_{1}(x)$ which have been before introduced as input from lattice data, will be calculated analytically within the same method, making finally the whole theory complete and selfconsistent. The first attempts in this direction have been already done in [6], where $\mu=1 / \mathrm{Tg}$ was calculated in terms of string tension. 


\section{Dynamics of the temperature phase transition in QCD}

Calculations of the free energy of gluons and quarks, which have been done in section 4, will serve us in this section to compute the phase transition temperature and to define the physical picture of the phase transition - in the first approximation of $1 / N_{c}$ expansion - which will be a very realistic picture. .

We shall consider two possible phases - the deconfined one at $T>T_{c}$ and the confined phase at $T<T_{c}$. The choice of the phase from physical point of view is dictated by the minimal value of the free energy (or the maximal value of pressure). The most important ingredient in the QCD free energy (which is absent in QED) is the nonperturbative energy density, connected to the gluonic condensate

$$
\varepsilon=\frac{\beta\left(\alpha_{s}\right)}{16 \alpha_{s}}\left\langle F_{\mu \nu}^{a} F_{\mu \nu}^{a}\right\rangle \simeq-\frac{11}{3} N_{c} \frac{\alpha_{s}}{16 \pi}\left\langle E^{a} E^{a}+H^{a} H^{a}\right\rangle
$$

Thus one write the free energy in the low phase (confined) as

$$
\frac{1}{V_{3}} F_{\text {low }}=\varepsilon-T \sum_{k} \frac{\left(2 m_{k} T\right)^{3 / 2}}{8 \pi^{3 / 2}} e^{-m_{k} / T}-\frac{\pi^{2}}{30} T^{4}+O\left(1 / N_{c}\right)
$$

where $m_{k}$ are massive particles (not pions), pions are given by the third term on the r.h.s. of (114).

For deconfined phase one should specify the condensate term (113). Since confinement is electric, deconfinement would logically imply disappearence of the electric part of the condensate $\left\langle E^{a} E^{a}\right\rangle$ in (113). However the NP part of $D_{1}(x)$ does not confine and therefore in $\left\langle E^{a} E^{a}\right\rangle=D^{E}(0)+D_{1}(0)$ the part $D_{1}^{E}(0)$ could survive. However lattice calculations of the Pisa group [42] imply, that both $D^{E}(0)$ and $D_{1}^{E}(0)$ vahish near $T=T_{c}$, while the magnetic part $D^{B}(0)+D_{1}^{B}(0)$ stay intact and contribute around one half of the total condensate, as it is for zero temperature.

Hence one can write the free energy for the high phase as

$$
\frac{1}{V_{3}} F_{h i g h}=\frac{1}{2} \varepsilon-\left(N_{c}^{2}-1\right) \frac{T^{4} \pi^{2}}{45}-\frac{7 \pi^{2}}{180} N_{c} T^{4} n_{f}
$$

where $n_{f}$ is the number of flavours, and the second and the third term refer to gluons and quarks computed respectively in (54) and (70).

Comparing (114) and (115) one can see that $F_{\text {high }}$ starts higher than $F_{\text {low }}$ at $T=0$ (note that $\varepsilon<0$ ) but drops faster and at some $T=T_{c}$ both $F$ are equal, which means that the phase transition takes place and nature chooses that phase which ensures lower free energy (higher pressure) (this is a consequence of the 2nd thermodynamic law).

This value of $T_{c}$ is [17, 18.

$$
T_{c}=\left[\frac{\frac{11}{6} N_{c} \frac{\alpha_{s}\langle F\rangle}{32 \pi}}{\frac{\pi^{2}}{45}\left(N_{c}^{2}-1\right)+\frac{7 \pi^{2}}{180} N_{c} n_{f}}\right]^{1 / 4} .
$$


Note that at large $N_{c}$ the critical temperature is $O\left(N_{c}^{0}\right)$, which is expected, but no other model predicts this correct dependence.

It is instructive to compare the values of $T_{c}$ from (116) and from recent lattice calculations [71].

Table 6: Values of $T_{c}$ for $N_{c}=3$ and different $n_{f}$ compared to lattice data [71]

\begin{tabular}{|l|l|l|}
\hline$S U(3)$ & $T_{c}\left(m_{\rho}=0.77\right)(\mathrm{MeV})$ & $\mathrm{Eq} \cdot(\mathbb{1 1 6}) \mathrm{MeV}$ \\
\hline$n_{f}=0^{*)}$ & 270 & $220-240$ \\
$n_{f}=2$ & 170 & 150 \\
$n_{f}=4$ & 131 & 134 \\
\hline
\end{tabular}

*) For $n_{f}=0$ the quenched value of the gluonic condensate was taken 3-4 times larger than the standard (nonquenched) value $\frac{\alpha_{s}}{\pi}\left\langle F^{2}\right\rangle=0.012 \mathrm{GeV}^{4}$. This in agreement with arguments of SVZ[2] and lattice calculations of Di Giacomo et al [42].

Several comments are in order. First, values of $T_{c}$ are surprisingly close to the lattice calculations which supports our picture of phase transition. Secondly, magnetic condensate is as strong as for $T=0$ (and becomes stronger with grouth of $T$ ). This leads to the spacial correlations above $T_{c}$, calculated in [20] and measured on the lattice.

Finally, one should not confuse the above physically correct picture of phase transition with a popular model, where all system is immerged in the huge bag with the standard bag pressure (which is order of magnitude smaller than gluonic condensate [2]). This latter picture leads to many inconsistencies and cannot be considered as a serious suggestion.

Recent development of our picture demonstrating a good description of nonperturbative dynamics of quark gluon plasma was done for $S U(2)$ in 21] and quite recently for $S U(3)$ in $[72]$. Further work incorporating both electric field in Polyakov lines and magnetic field modifying gluon and quark free energy is now under investigation.

\section{Acnowledgements}

The author is grateful to the Organizing Commitee of the International School for a kind hospitality and good creative atmosphere during the whole period.

\section{References}

[1] H.Lehmann, K.Symanzik, W.Zimmermann, Nuovo Cim. 1 (1955) 205

[2] M.A.Shifman, A.I.Vainshtein and V.I.Zakharov, Nucl. Phys. B147 (1979) 385 
[3] V.N.Gribov, Nucl. Phys. B139 (1978) 1

[4] T.D.Lee, Particle Physics and Introduction to Field Theory, Harwood, Chur.

[5] Yu.A.Simonov, Sov. J. Nucl. Phys. 41 (1985) 835

[6] Yu.A.Simonov, Phys. At. Nucl. 61 (1998) 855; Yad. Fiz. 61 (1989) 941

[7] Yu.A.Simonov, in: Lecture Notes in Physics, v.479, p.144, Springer, 1996

[8] Yu.A.Simonov, Phys. At. Nucl. 58 (1995) 107 (Yad. Fiz. 58 (1995) 113)

[9] Yu.A.Simonov, Nucl. Phys. B307 (1988) 512

[10] Yu.A.Simonov and J.A.Tjon, Ann Phys. 228 (1993) 1

[11] J.Schwinger, Phys. Rev. 80 (1951) 664

[12] R.Feynman, Phys. Rev. 80 (1950) 440

[13] G.A.Milekhin and E.S.Fradkin, ZhETF 45 (1963) 1926;

E.S.Fradkin, Trudy FIAN 29 (1965) 7;

M.B.Halpern , A.Jevicki, P.Senjanovic, Phys. Rev. D16 (1977) 2476;

R.Brandt et.al. Phys. Rev. D19 (1979) 1153;

S.Samuel, nucl. Phys. B149 (1979) 517;

J.Ishida and A.Hosoya, Progr. Theor. Phys. 62 (1979) 544

[14] Z.Bern, D.A. Kosower, Phys. Rev. Lett. 66 (1991) 1669; Nucl. Phys. B379 (1992) 451;

Z.Bern, D.C.Dunbar, Nucl. Phys. B379 (1992) 562

[15] M.J.Strassler, Nucl. Phys. 385 (1992) 145

[16] Yu.A.Simonov, Phys. Lett. B464 (1999) 265

[17] Yu.A.Simonov, Phys. At. Nucl. 58 (1995) 309

[18] Yu.A.Simonov, JETP Lett. 54 (1991) 249; JETP Lett. 55 (1992) 637

[19] Yu.A.Simonov, in "Varenna 1995, selected topics in nonperturbative QCD", 319-337

[20] E.L.Gubankova and Yu.A,Simonov, Phys. Lett. B360 (1995) 93

[21] H.G.Dosch, H.J.Pirner and Yu. A.Simonov, Phys. Lett. B349 (1995) 335

[22] N.G.Van Kampen, Phys. Rep. C24 (1976) 171; Physica 74 (1974) 215

[23] Yu.A.Simonov, Yad. Fiz. 54 (1991) 192 
[24] Yu.A.Simonov, Z. Phys. C53 (1992) 419

[25] Yu.A.Simonov, JETP Lett. 57 (1993) 525

[26] A.M.Badalian and Yu.A.Simonov, Yad. Fiz, 60 (1997) 714

[27] D.J.Gross, R.D.Pisarski, L.G.Yaffe, Rev. Mod. Phys. 53 (1981) 43

[28] Yu.A.Simonov, Nucl. Phys. B324 (1989) 67

[29] M.Halpern, Phys. Rev. D19 (1979) 517;

I.Aref'eva, Theor Math. Phys. 43 (1980) 353;

N.Bralic, Phys. Rev. D22 (1980) 3090;

Yu.A.Simonov, Sov J. Nucl. Phys. 50 (1989) 134;

M.Hirayama, S.Matsubara, hep-th/9712120

[30] S.V.Ivanov, G.P.Korchensky, Phys. Lett. B154 (1985) 197;

S.V.Ivanov, G.P.Korchensky, A.V.Radyushkin, Sov.Y. Nucl. Phys. 44 (1986) 145 .

[31] V.I.Shevchenko, Yu.A.Simonov, Phys. Lett. B437 (1998) 146;

L.Lukaszuk, hep-th/9611192

[32] H.G.Dosch and Yu. A.Simonov, Phys. Lett. B205 (1988) 339

[33] L.Del. Debbio, A.Di. Giacomo, and Yu. A.Simonov, Phys. Lett. B332 (1994) 111

[34] G.Bali, hep-lat/9908021; hep-lat/0006022

[35] V.I.Shevchenko, Yu.A.Simonov, Phys. Rev. Lett. 85 (2000) 1811; hep$\mathrm{ph} / 0001299$

[36] H.B.G.Casimir, Proc.Roy.Acad. Amsterdam 34 (1931) 844.

[37] J.A. de Azcárraga, et al, Nucl.Phys.B510 (1998) 657.

[38] M.Peter, Phys. Rev. Lett. 78 (1997) 602, Nucl. Phys. B501 471 (1997);

Y.Schroeder, Phys. Lett. B447 321 (1999)

[39] N.A.Campbell, I.H.Jorysz, C.Michael, Phys. Lett. B167 (1986) 91

C.Michael, Nucl. Phys.Proc. Suppl.) 26 (1992) 417

C.Michael, hep-ph/9809211

S.Deldar, hep-lat/9809137

[40] M.Lüscher, K.Symanzik, P.Weisz, Nucl.Phys.B173 (1980) 365. 
[41] M.Campostrini, A.Di Giacomo and G.Mussardo, Z.Phys. C25 (1984) 173;

A.Di Giacomo and H.Panagopoulos, Phys. Lett. B285 (1992) 133;

A.Di Giacomo, E.Meggiolaro and H.Panagopoulos, hep-lat/9603097

[42] A.Di Giacomo, E.Meggiolaro and H.Panagopoulos, Nucl. Phys. B483 (1997) 371 ;

A.Di Giacomo, M.D'elia, H.Panagopoulos, E.Meggiolaro, hep-lat/9808056

[43] M.Foster and C.Michael, Phys. Rev. D49 (1999) 094509, hep-lat/9811010

[44] Yu.A.Simonov, Nucl. Phys. B (in press), hep-ph/0003114

[45] V.I.Shevchenko, Yu.A.Simonov, Phys. Lett. B437 (1998) 131; hep-ph/9803377

[46] A.M.Badalian and Yu.A.Simonov, Phys. At. Nucl. 59 (1996) 2164

[47] E.Eichten and F.Feinberg, Phys. Rev. D23 (1981) 2724

[48] A.M.Badalian and V.P.Yurov, Yad. Fiz. 51 (1990) 1368; ibid 56 (1993)

[49] A.M.Badalian and V.l. Morgunov, Yad. Fiz. 62 (1999)1086 or Phys. At. Nucl. 62 (1999) 1019

[50] A.M.Badalian and V.l. Morgunov, Phys. Rev. D60 (1999) 116008; hepph/9901430

[51] A.M.Badalian and B.L.G.Bakker,hep-ph/0004021; Phys. Rev. D62 (2000) 94031

[52] Yu.A.Simonov, S.Titard and F.J.Yndurain, Phys. Lett. B354 (1995) 435

[53] V.L.Morgunov, V.I.Shevchenko and Yu.A.Simonov, Phys. At. Nucl. 61 (1998) 664, Yad. Fiz. 61 (1998) 739

[54] V.L.Morgunov, V.I.Shevchenko and Yu.A.Simonov, hep-ph/9704282, Phys. Lett. B416 (1998) 433

[55] Yu.A,Simonov, Phys. At Nucl. 60 (1997) 2069, hep-ph/9704301

[56] Yu.A,Simonov, Phys. At Nucl. 62 (1999) 1932, hep-ph/9912484

[57] A.M.Polyakov, Gauge fields and strings, Harwood Academic, 1987;

A.Yu.Dubin, JETP Lett. 56 (1992) 545;

Yu.S.Kalashnikova, A.V,Nefediev, Phys. At. Nucl. 60 (1997) 1389; 61 (1998) 785

[58] A.Yu.Dubin, A.B.Kaidalov and Yu.A.Simonov, Phys. Lett. B323 (1994) 41 
[59] D.R.Stanly and D.Robson, Phys. Lett. B45 (1980) 235;

P.Cea, G.nardulli and G.Preparata, Z.Phys. C16 (1982) 135;

J.Calson et al. Phys. Rev. D27 (1983) 233;

N.Isgur and S.Godfrey, Phys. Rev. D32 (1985) 189;

J.L.Basdevant, S.Boukraa, Z.Phys. C28 (1983) 413.

[60] Yu.A.Simonov, Phys. Lett. B226 (1989) 151, for a comprehensive review see 23

[61] Yu.A.Simonov, QCD and Topics in Hadron Physics, Lectures at the XVII International School of Physics, Lisbon, 29 September -4 October, hep-ph/9911237 (to appear in Proceedings)

[62] Yu.S.Kalashnikova and A.V.Nefediev, hep-ph/0008242, Phys. Lett. B (in press)

[63] Yu.A.Simonov, Phys. Lett. B228 (1989) 413

[64] M.Fabre dela Ripelle and Yu.A.Simonov, Ann. Phys. 212 (1991) 235

[65] B.O.Kerbikov and Yu.A.Simonov, Phys. Rev. D; hep-ph/0001243;

B.O.Kerbikov, Phys. At. Nucl.(in press); hep-ph/0003209

[66] Yu.A.Simonov, Yad. Fiz. 3 (1966) 630;

A.M.Badalian and Yu.A.Simonov, Yad. Fiz. 3 (1966) 1032;

F.Calogero and Yu.A.Simonov, Phys. Rev. 183 (1968) 869

[67] S.Capstick and N.Isgur, Phys. Rev. D34 (1986) 2809

[68] D.S.Kuzmenko and Yu.A.Simonov, Phys. Lett. B (in press); hep-ph/0006192

[69] A.B.Kaidalov and Yu.A.Simonov, Phys. Lett. B477 (2000) 163

[70] A.B.Kaidalov and Yu.A.Simonov, Yad. Fiz.; hep-ph/9911291

[71] F.Karsch, hep-lat/9909006

[72] N.O.Agasyan (in preparation) 